\title{
Leptogenesis in crossing and runaway regimes
}

\section{A. Hohenegger ${ }^{a}$ and A. Kartavtsev ${ }^{b}$}

${ }^{a}$ Institute for Mathematics and Natural Sciences, University of Stavanger, Kjell Arholms gate 41, 4036 Stavanger, Norway

${ }^{b}$ Theoretical Physics Division, Max-Planck-Institut für Physik, Föhringer Ring 6, 80805 München, Germany

E-mail: andreas.hohenegger@uis.no, alexander.kartavtsev@mpp.mpg.de

ABSTRACT: We study the impact of effective thermal masses and widths on resonant leptogenesis. We identify two distinct possibilities which we refer to as 'crossing' and 'runaway' regimes. In the runaway regime the mass difference grows monotonously with temperature, whereas it initially decreases in the crossing regime, such that the effective masses become equal at some temperature. Following the conventional logic the source of the asymmetry would vanish in the latter case. Using non-equilibrium quantum field theory, we analytically demonstrate that the vanishing of the difference of the effective masses does however neither imply a suppression nor a strong enhancement of the source for the lepton asymmetry. In the vicinity of the crossing point the asymmetry calculated in an (improved) Boltzmann limit develops a spurious peak, which signals the breakdown of the quasiparticle approximation. In the exact result this spurious enhancement is compensated by coherent transitions between the two mass shells. Despite the breakdown of the quasiparticle approximation off-shell contributions remain negligibly small even at the crossing point.

KEYworDs: Thermal Field Theory, Cosmology of Theories beyond the SM

ARXIV EPRINT: 1404.5309 


\section{Contents}

1 Introduction $\quad 1$

2 Setup 4

3 Fundamental symmetries and dynamics $\quad 16$

$4 \quad$ Runaway and crossing regimes $\quad 22$

5 Analytical treatment of leading effects 26

$6 \quad$ Numerical treatment of sub-leading effects 33

$\begin{array}{lll}7 & \text { Summary and outlook } & 36\end{array}$

\section{Introduction}

From the theoretical point of view the baryogenesis via leptogenesis scenario [1] is a very attractive explanation for the observed baryon asymmetry of the Universe. One of its key ingredients are heavy Majorana neutrinos, which may cause a lepton asymmetry to emerge. The generation of the asymmetry can proceed via $C P$-violating decays and inverse decays of the heavy neutrinos [1], their $C P$-violating oscillations [2], or via a combination of the two. The first case is typically realized for Majorana neutrinos with masses considerably larger than the sphaleron freeze-out temperature. This possibility has been explored extensively using the usual Boltzmann-like equations with decay and scattering amplitudes computed using methods of zero temperature [3-14] or thermal [15-18] quantum field theory. The second case is typically realized for Majorana neutrinos with masses below the sphaleron freeze-out temperature. It has been studied using the 'density matrix formalism' [19-27] which was originally developed in [28] and cross-checked in an alternative approach [29].

Recently, various aspects of leptogenesis have been re-analysed using the first-principle Kadanoff-Baym formalism [30-33] as well as self-consistent Boltzmann-like [34-39] and quantum-kinetic [40-43] equations systematically derived from the former. A lot of effort has been put into the analysis of the phenomenologically particularly interesting scenario of resonant leptogenesis [44-52]. Resonant leptogenesis is realized for a quasi-degenerate mass spectrum of the heavy neutrinos, when the difference of the masses is comparable to the sum of the decay widths. In this domain of the parameter space the $C P$-violating parameters are resonantly enhanced. In the case of leptogenesis via $C P$-violating decays and inverse decays this allows one to lower the Majorana masses down to the TeV scale $[50,51]$ which is in principle accessible at the LHC [53-58]. In case of leptogenesis via $C P$-violating 
oscillations of the Majorana neutrinos their masses can be as low as a few $\mathrm{GeV}$ [19-26], such that they can be searched for in dedicated high-intensity experiments [59].

The regime of resonant asymmetry generation is not only of particularly high interest but presumably also the most difficult one to study. Early works using the Boltzmann equation and relying on the zero temperature quantum field theory concentrated on the derivation of $C P$-violating parameters $\epsilon_{i}$. In case of two generations of Majorana neutrinos it has been found in [52], using the on-shell renormalization scheme, that in the basis where the mass matrix is diagonal:

$$
\epsilon_{i} \propto \frac{M_{j}^{2}-M_{i}^{2}}{\left(M_{j}^{2}-M_{i}^{2}-\frac{1}{\pi} \ln \left(M_{j}^{2} / M_{i}^{2}\right)\right)^{2}+\left(M_{j} \Gamma_{j}-M_{i} \Gamma_{i}\right)^{2}} .
$$

In the limit of vanishing mass difference, $M_{2}=M_{1}$, the numerator of (1.1) becomes zero whereas the denominator does not if $\Gamma_{2} \neq \Gamma_{1}$. The vanishing of the $C P$-violating parameters is required in this limit, because the corresponding Lagrangian is $C P$-invariant [60, 61]. On the other hand, if $M_{2} \rightarrow M_{1}$ and $\Gamma_{2} \rightarrow \Gamma_{1}$ simultaneously then, according to the above expression, the $C P$-violating parameters do not vanish even though the Lagrangian is $C P$-invariant as well in this case. The origin of this problem lies in the use of the quasiparticle picture built-in in the Boltzmann approximation. The very fact that the peaks of the spectral functions that correspond to the quasiparticle excitations strongly overlap in the resonant regime renders the use of Boltzmann equations problematic. The question which behaviour the $C P$-violating parameter exhibits in this 'doubly degenerate' limit has recently been answered in [62], using the formalism of non-equilibrium quantum field theory and without invoking the quasiparticle approximation. It has been found that, for $M_{2} \rightarrow M_{1}$ and $\Gamma_{2} \rightarrow \Gamma_{1}$, the regulator $M_{j} \Gamma_{j}-M_{i} \Gamma_{i}$ in the denominator of (1.1) is effectively replaced by $M_{j} \Gamma_{j}+M_{i} \Gamma_{i}$ due to additional contributions that describe coherent transitions between the Majorana neutrino species. This implies that in the resonant regime both $C P$-violating (inverse) decays and oscillations play an important role and must be taken into account in a self-consistent analysis.

According to the conventional analysis the $C P$-violating parameters take their maxima if the mass difference is of the order of the sum of the decay widths. However the early Universe expands and cools rapidly. During the time interval in which most of the asymmetry is generated, the temperature (measured in units of the heavy neutrino mass) can drop substantially. In the favoured regime, thermal corrections to the effective masses can be comparable to the mass difference itself. Depending on the values of the couplings there are two possibilities, see figure 1 (left). In the runaway regime the mass difference grows with increasing temperature, whereas in the crossing regime the difference of the masses initially decreases such that the two masses become equal at some temperature, and then increases again with increasing temperature. In this work we study the influence of thermal corrections to the masses in these two regimes, which have not been considered before, on resonant leptogenesis. To this end we consider a simple toy-model which proved to be useful in the past for the analysis of leptogenesis [18, 34, 35, 61, 63]. Throughout this work we emphasize the strict requirement that the obtained source terms for the lepton 
Effective masses, $\mathrm{R}=1$

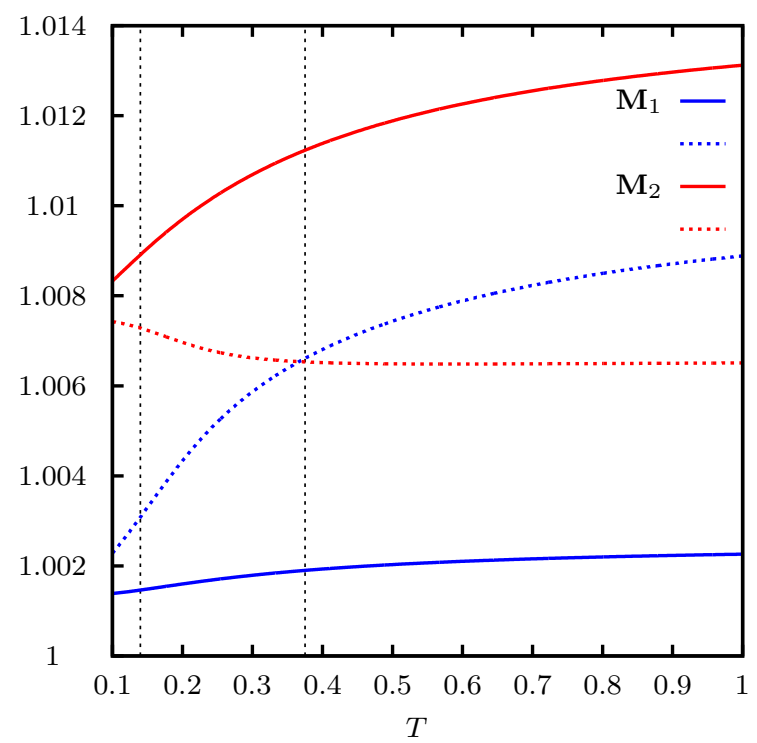

Effective measure of $\mathrm{CP}$-violation, $\mathrm{R}=1$

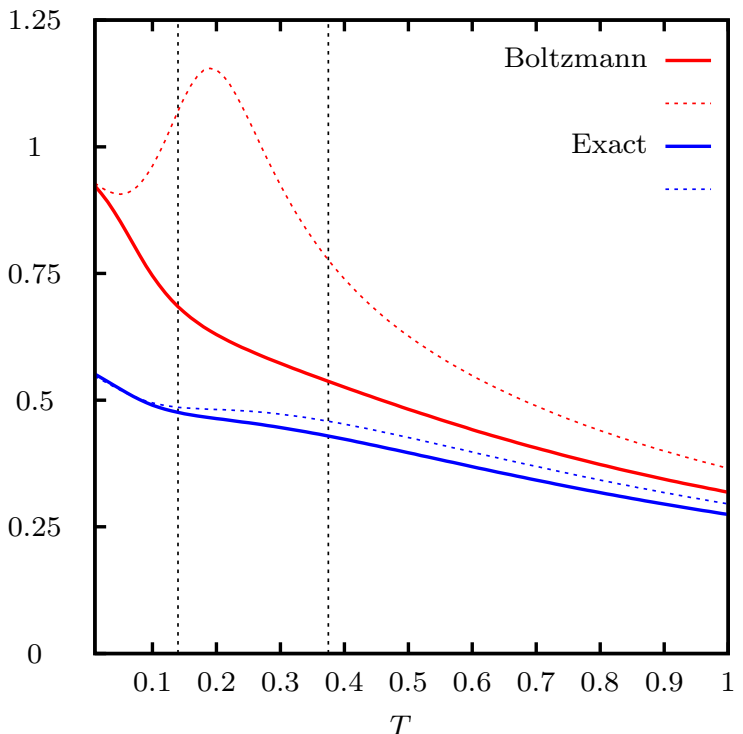

Figure 1. Sketch of the dependence of the effective masses (left) and effective measure of $C P$-violation (right) in the runaway (solid lines) and crossing (dotted lines) regimes for a quasidegenerate mass spectrum of the heavy particles. $R$ denotes the resonance parameter which quantifies the mass splitting in vacuum (see below).

asymmetry need to respect the $C P$-properties of the underlying theory represented by the Lagrangian as the parameters of corresponding quasiparticles (insofar they can be defined) evolve due to medium effects.

In order to be able to study the interplay of coherent oscillations and resonant enhancement rigorously we also adopt a simplified physical picture [31-33, 62]. We neglect the expansion of the Universe and assume instead that the toy-Majorana neutrinos are coupled through their decays to a thermal bath composed of the decay products (toy-leptons). The deviation from thermal equilibrium needed to produce an asymmetry is induced by an instantaneous perturbation. This setting may differ from the conventional physical picture connected to standard cosmology but allows us to obtain analytic solutions and study the source of the asymmetry generation rigorously from first principles, in terms of statistical propagators and spectral functions.

Before we present the qualitatively interesting results obtained in this approach let us discuss what one would naively expect from the vacuum expression (1.1) in both regimes. Because in the runaway regime the difference of the effective masses grows with temperature, one could expect that the overlap of the peaks of the spectral function decreases simultaneously. Therefore, the quality of the quasiparticle approximation can be expected to improve. The growing mass difference is also expected to result in a smaller $C P$-violation. On the other hand, because in the crossing regime the difference of the effective masses vanishes at some temperature, the peaks of the spectral function are expected to overlap at this point. This suggests a complete breakdown of the quasiparticle approximation and large relative size of off-shell contributions. Furthermore, from (1.1) one could also expect 
that the $C P$-violating parameters vanish, or are at least suppressed at the crossing point. At even higher temperatures the mass difference grows again and the applicability of the quasiparticle approximation may be expected to be restored. The same logic would imply that in the $M_{j}=M_{i}$ case $\epsilon_{i}$ vanishes at zero temperature but would in general evolve into a finite one as the temperature is increased.

The results of the first-principles analysis, see figure 1 (right), show that this naive picture is only partially correct. In the runaway regime we observe that the result obtained in the Boltzmann approximation slowly approaches the exact one at high temperatures. This signals that the quality of the quasiparticle approximation indeed improves at high temperatures. In agreement with the expectations the effective measure of $C P$-violation decreases monotonously with increasing temperature. In the crossing regime the quasiparticle approximation breaks down at the crossing point. As can be inferred from figure 1 (right), at the crossing temperature the $C P$-violating source computed in an improved Boltzmann approximation develops a spurious peak which is absent in the exact result. On the other hand, the expected vanishing (or at least suppression) of the source at the crossing point does not take place.

The outline of the paper is as follows. In section 2 we present the toy-model and derive an equation for the asymmetry in the framework of non-equilibrium quantum field theory. In section 3 we analytically demonstrate that the asymmetry automatically vanishes if both the Lagrangian and initial conditions are $C P$-symmetric. The analysis of the effective masses and widths as well as of the behaviour of the spectral function is carried out in section 4. In section 5 we present analytical estimates of the leading contributions to the effective measure of $C P$-violation. Numerical estimates of the size of the sub-leading contributions are given in section 6 . We summarize the main results in the beginning of each section. Finally, in section 7 we conclude and give a qualitative explanation for the difference between the naively expected behaviour and the exact results.

\section{Setup}

In this section we derive an equation for the asymmetry in the framework of non-equilibrium quantum field theory. The derivation closely follows the analysis which was performed in [62] and recently generalized to the case of expanding universe in [64,65]. We also establish a connection between this first-principle approach and the commonly used Boltzmann approximation. In addition we demonstrate that in the Boltzmann approximation the test solution that we use corresponds to the weak washout regime and describes free decay of the heavy particles.

Model. To reduce the technical complications to a minimum and yet to include all qualitatively important effects for the generation of the asymmetry we use a simple toy model studied previously in $[18,34,35,61,63]$. The model contains one complex and two real scalar fields:

$$
\mathcal{L}=\frac{1}{2} \partial^{\mu} \psi_{i} \partial_{\mu} \psi_{i}-\frac{1}{2} \psi_{i} M_{i j}^{2} \psi_{j}+\partial^{\mu} \bar{b} \partial_{\mu} b-m^{2} \bar{b} b-\frac{\lambda}{2 ! 2 !}(\bar{b} b)^{2}-\frac{h_{i}}{2 !} \psi_{i} b b-\frac{h_{i}^{*}}{2 !} \psi_{i} \bar{b} \bar{b},
$$


where $\bar{b}$ denotes the complex conjugate of $b$. Here and in the following we assume summation over repeated indices, unless otherwise specified. Despite its simplicity, the model incorporates all features relevant for leptogenesis. The real scalar fields imitate the (two lightest) heavy right-handed neutrinos, whereas the complex scalar field models the leptons. The U(1) symmetry, which we use to define "lepton" number, is explicitly broken by the presence of the last two terms, just as the $B-L$ symmetry is explicitly broken by Majorana mass terms in phenomenological models. Thus the first Sakharov condition is fulfilled. The couplings $h_{i}$ model the complex Yukawa couplings of the right-handed neutrinos to leptons and the Higgs. By rephasing the complex scalar field at least one of the couplings $h_{i}$ can be made real. If $\arg \left(h_{1}\right) \neq \arg \left(h_{2}\right)$ the other one remains complex and there is $C$-violation, as is required by the second Sakharov condition. Note that, in the scalar toy model, $C P$-transformations on the fields are identical to $C$-transformations up to the sign change of the spatial coordinates.

Non-equilibrium quantum field theory approach. As can be inferred from (2.1) the Noether current of the complex field is given by

$$
j_{\mu}=2 i\left[\bar{b}(x) \partial_{\mu_{x}} b(x)-b(x) \partial_{\mu_{x}} \bar{b}(x)\right]=2 i \lim _{y \rightarrow x}\left[\partial_{\mu_{x}} \bar{b}(y) b(x)-\partial_{\mu_{y}} b(x) \bar{b}(y)\right],
$$

where $b$ and $\bar{b}$ are field operators in the Heisenberg representation. The expectation value of the current with respect to the initial state is

$$
J_{\mu}(x)=\left\langle j_{\mu}(x)\right\rangle=2 i \lim _{y \rightarrow x}\left[\partial_{\mu_{x}} D_{<}(x, y)-\partial_{\mu_{y}} D_{>}(x, y)\right]
$$

where

$$
D_{>}(x, y) \equiv\langle b(x) \bar{b}(y)\rangle=\operatorname{Tr}[\mathscr{P} b(x) \bar{b}(y)], \quad D_{<}(x, y) \equiv\langle\bar{b}(y) b(x)\rangle=\operatorname{Tr}[\mathscr{P} \bar{b}(y) b(x)],
$$

are so-called Wightmann two-point functions. In general, $D_{\gtrless}$ are complex-valued. Using the hermiticity of the density matrix $\mathscr{P}$ and cyclic invariance of the trace we find that they satisfy [34]

$$
D_{>}^{*}(x, y)=D_{>}(y, x), \quad D_{<}^{*}(x, y)=D_{<}(y, x) .
$$

Instead of the Wightmann two-point functions one frequently uses the spectral function and statistical propagator:

$$
D_{\gtrless}(x, y)=D_{F}(x, y) \mp \frac{i}{2} D_{\rho}(x, y) .
$$

As can be inferred from (2.4) and (2.6), they are defined as

$$
D_{F}(x, y) \equiv \frac{1}{2}\langle\{b(x), \bar{b}(y)\}\rangle, \quad D_{\rho}(x, y) \equiv i\langle[b(x), \bar{b}(y)]\rangle,
$$

where the square brackets denote the commutator and the curly ones denote the anticommutator of the fields. Using (2.5) we find that under complex conjugation they transform as

$$
D_{F}^{*}(x, y)=D_{F}(y, x), \quad D_{\rho}^{*}(x, y)=-D_{\rho}(y, x)
$$


Substituting (2.6) into (2.3) we obtain

$$
J_{\mu}(x)=2 i \lim _{y \rightarrow x}\left(\partial_{\mu_{x}}-\partial_{\mu_{y}}\right) D_{F}(x, y)-\lim _{y \rightarrow x}\left(\partial_{\mu_{x}}+\partial_{\mu_{y}}\right) D_{\rho}(x, y) .
$$

The definition of the spectral function, eq. (2.7), combined with the canonical equal-time commutation relations,

$$
[b(t, \mathbf{x}), \dot{\bar{b}}(t, \mathbf{y})]=[\bar{b}(t, \mathbf{x}), \dot{b}(t, \mathbf{y})]=i \delta(\mathbf{x}-\mathbf{y}),
$$

then implies that the spectral function does not contribute to the current. The divergence of the current,

$$
\partial^{\mu} J_{\mu}(x)=i \lim _{y \rightarrow x}\left(\partial^{\mu_{x}}+\partial^{\mu_{y}}\right)\left(\partial_{\mu_{x}}-\partial_{\mu_{y}}\right) D_{F}(x, y)=i \lim _{y \rightarrow x}\left(\square_{x}-\square_{y}\right) D_{F}(x, y),
$$

can be rewritten using the Kadanoff-Baym equations (KBE) for the complex field. For Gaussian initial conditions the latter take the form [34]

$$
\begin{aligned}
& {\left[\square_{x}+m^{2}\right] D_{F}(x, y)=\int_{t_{0}}^{y^{0}} d^{4} z \Sigma_{F}(x, z) D_{\rho}(z, y)-\int_{t_{0}}^{x^{0}} d^{4} z \Sigma_{\rho}(x, z) D_{F}(z, y),} \\
& {\left[\square_{x}+m^{2}\right] D_{\rho}(x, y)=\int_{x^{0}}^{y^{0}} d^{4} z \Sigma_{\rho}(x, z) D_{\rho}(z, y),}
\end{aligned}
$$

where $t_{0}$ is the initial time surface and $\Sigma_{F(\rho)}$ are the statistical (spectral) components of the self-energy. Substituting (2.12a) into (2.11) we obtain

$$
\begin{array}{r}
\partial^{\mu} J_{\mu}(x)=-i \int_{t_{0}}^{x^{0}} d z^{0} \int d^{3} z\left[\Sigma_{\rho}(x, z) D_{F}(z, x)-\Sigma_{F}(x, z) D_{\rho}(z, x)\right. \\
\left.+D_{F}(x, z) \Sigma_{\rho}(z, x)-D_{\rho}(x, z) \Sigma_{F}(z, x)\right] .
\end{array}
$$

For a spatially homogeneous system $\partial^{\mu} J_{\mu}=\partial^{0} J_{0}=\dot{q}(t)$, where $q$ is the charge density. Using furthermore (2.8) and similar relations for the self-energies we can simplify (2.13) to

$$
\begin{aligned}
\dot{q}(t) & \equiv S(x)-W(x) \\
& =2 \int_{t_{0}}^{t} d z^{0} \int d^{3} z \operatorname{Im}\left[\Sigma_{\rho}(x, z) D_{F}(z, x)-\Sigma_{F}(x, z) D_{\rho}(z, x)\right] .
\end{aligned}
$$

This expression gives an exact result for the time derivative of the asymmetry (assuming Gaussian initial conditions) and provides the basis for various approximation schemes, e.g. the Boltzmann approximation.

The source and washout terms in (2.14) are defined by

$$
S(x) \equiv 2 \int_{t_{0}}^{x^{0}} d z^{0} \int d^{3} z\left[\operatorname{Im} \Sigma_{\rho}(x, z) \operatorname{Re} D_{F}(z, x)-\operatorname{Im} \Sigma_{F}(x, z) \operatorname{Re} D_{\rho}(z, x)\right],
$$


and by

$$
W(x) \equiv-2 \int_{t_{0}}^{x^{0}} d z^{0} \int d^{3} z\left[\operatorname{Re} \Sigma_{\rho}(x, z) \operatorname{Im} D_{F}(z, x)-\operatorname{Re} \Sigma_{F}(x, z) \operatorname{Im} D_{\rho}(z, x)\right],
$$

respectively. The definition of the washout term, which should describe the washout of a present asymmetry, is motivated by the following considerations. The operation of charge conjugation replaces the fields in (2.4) by their complex conjugates, see section 3 for more details, and the density matrix by the charge conjugate one:

$$
\begin{aligned}
& D_{>}(x, y) \rightarrow C D_{>}(x, y) C^{-1}=\operatorname{Tr}\left[\mathscr{P}^{c} \bar{b}(x) b(y)\right]=D_{<}^{c}(y, x)=D_{<}^{c *}(x, y), \\
& D_{<}(x, y) \rightarrow C D_{<}(x, y) C^{-1}=\operatorname{Tr}\left[\mathscr{P}^{c} b(y) \bar{b}(x)\right]=D_{>}^{c}(y, x)=D_{>}^{c *}(x, y),
\end{aligned}
$$

where we have used relations (2.5) in the last equalities of (2.17a) and (2.17b). Combining (2.6) and (2.17) we find that the $C$-conjugated statistical propagator and spectral function are given by

$$
D_{F(\rho)}(x, y) \rightarrow C D_{F(\rho)}(x, y) C^{-1}=D_{F(\rho)}^{c *}(x, y) .
$$

In a $C$-symmetric configuration $\mathscr{P}^{c}=\mathscr{P}$ and therefore the statistical propagator as well as the spectral function are real-valued in this case. This implies that, in agreement with physical considerations, the washout term, which is proportional to the imaginary part of the propagators, vanishes in a $C$-symmetric configuration. Let us now consider the source term. To this end we need to specify the form of the self-energies. At one-loop level they read [35]

$$
\begin{aligned}
& \Sigma_{F}(x, y)=-H_{i j}^{*}\left[G_{F}^{i j}(x, y) D_{F}(y, x)+\frac{1}{4} G_{\rho}^{i j}(x, y) D_{\rho}(y, x)\right], \\
& \Sigma_{\rho}(x, y)=+H_{i j}^{*}\left[G_{F}^{i j}(x, y) D_{\rho}(y, x)-G_{\rho}^{i j}(x, y) D_{F}(y, x)\right],
\end{aligned}
$$

where we have introduced $H_{i j} \equiv h_{i} h_{j}^{*}$. The statistical and spectral propagators of the mixing fields are defined analogously to (2.7):

$$
G_{F}^{i j}(x, y)=\frac{1}{2}\left\langle\left\{\psi_{i}(x), \psi_{j}(y)\right\}\right\rangle, G_{\rho}^{i j}(x, y)=i\left\langle\left[\psi_{i}(x), \psi_{j}(y)\right]\right\rangle .
$$

From the definitions (2.20) it follows that

$$
G_{F}^{i j}(x, y)=G_{F}^{j i}(y, x), \quad G_{\rho}^{i j}(x, y)=-G_{\rho}^{j i}(y, x) .
$$

Furthermore, using the hermiticity of the density matrix $\mathscr{P}$ and the cyclic invariance of the trace, one can show that these matrices are real-valued. Therefore, in a $C$-symmetric configuration:

$$
S(x) \equiv-2 \operatorname{Im} H_{i j} \int_{t_{0}}^{x^{0}} d z^{0} \int d^{3} z\left[G_{F}^{i j}(x, z) \Pi_{\rho}(z, x)-G_{\rho}^{i j}(x, z) \Pi_{F}(z, x)\right],
$$


where we introduced

$$
\begin{aligned}
\Pi_{F}(z, x) & \equiv D_{F}^{s}(z, x) D_{F}^{s}(z, x)-\frac{1}{4} D_{\rho}^{s}(z, x) D_{\rho}^{s}(z, x), \\
\Pi_{\rho}(z, x) & \equiv 2 D_{F}^{s}(z, x) D_{\rho}^{s}(z, x),
\end{aligned}
$$

for notational convenience and the superscript ' $s$ ' refers to a $C$-symmetric configuration.

For spatially homogeneous systems the two-point functions depend only on the difference of the spatial coordinates, $\mathbf{s} \equiv \mathbf{x}-\mathbf{y}$, and it is convenient to introduce their partial Wigner-transforms,

$$
D_{F, \rho}\left(x^{0}, y^{0}, \mathbf{p}\right) \equiv \int d^{3} s e^{-i \mathbf{p s}} D_{F, \rho}\left(x^{0}, y^{0}, \mathbf{s}\right)
$$

The definitions for the self-energies are similar. Substituting (2.24) into (2.22) we obtain

$$
S(t)=-2 \operatorname{Im} H_{i j} \int_{t_{0}}^{t} d t^{\prime} \int \frac{d^{3} q}{(2 \pi)^{3}}\left[G_{F}^{i j}\left(t, t^{\prime}, \mathbf{q}\right) \Pi_{\rho}\left(t^{\prime}, t, \mathbf{q}\right)-G_{\rho}^{i j}\left(t, t^{\prime}, \mathbf{q}\right) \Pi_{F}\left(t^{\prime}, t, \mathbf{q}\right)\right] .
$$

Integrating the source term (2.25) over $t$ and using the identity

$$
\int_{t_{0}}^{t} d t^{\prime} \int_{t_{0}}^{t^{\prime}} d t^{\prime \prime}\left[f\left(t^{\prime}, t^{\prime \prime}\right)+f\left(t^{\prime \prime}, t^{\prime}\right)\right]=\int_{t_{0}}^{t} d t^{\prime} \int_{t_{0}}^{t} d t^{\prime \prime} f\left(t^{\prime}, t^{\prime \prime}\right)
$$

we obtain a 'symmetrized' expression for the charge density which would be generated in the absence of the washout processes:

$$
\begin{aligned}
q_{S}(t)= & -\operatorname{Im} H_{i j} \int_{t_{0}}^{t} d t^{\prime} \int_{t_{0}}^{t} d t^{\prime \prime} \int \frac{d^{3} q}{(2 \pi)^{3}} \\
& \times\left[G_{F}^{i j}\left(t^{\prime}, t^{\prime \prime}, \mathbf{q}\right) \Pi_{\rho}\left(t^{\prime \prime}, t^{\prime}, \mathbf{q}\right)-G_{\rho}^{i j}\left(t^{\prime}, t^{\prime \prime}, \mathbf{q}\right) \Pi_{F}\left(t^{\prime \prime}, t^{\prime}, \mathbf{q}\right)\right] .
\end{aligned}
$$

Taking furthermore into account that $\operatorname{Im} H_{i i}=0$ and using $\Pi_{F(\rho)}\left(t^{\prime \prime}, t^{\prime}, \mathbf{q}\right)= \pm \Pi_{F(\rho)}\left(t^{\prime}, t^{\prime \prime}, \mathbf{q}\right)$ as well as the properties (2.21) we finally arrive at

$$
\begin{aligned}
q_{S}(t)= & -2 \operatorname{Im} H_{12} \int_{t_{0}}^{t} d t^{\prime} \int_{t_{0}}^{t} d t^{\prime \prime} \int \frac{d^{3} q}{(2 \pi)^{3}} \\
& \times\left[G_{F}^{12}\left(t^{\prime}, t^{\prime \prime}, \mathbf{q}\right) \Pi_{\rho}\left(t^{\prime \prime}, t^{\prime}, \mathbf{q}\right)-G_{\rho}^{12}\left(t^{\prime}, t^{\prime \prime}, \mathbf{q}\right) \Pi_{F}\left(t^{\prime \prime}, t^{\prime}, \mathbf{q}\right)\right] .
\end{aligned}
$$

Equation (2.28) provides an exact result for the asymmetry in the limit in which washout processes can be neglected. Importantly, it does not rely on the quasiparticle approximation and can be used to study the off-shell and oscillation effects possibly relevant in the resonant regime. 
Equilibrium solution. To evaluate (2.28) we need explicit expressions for the offdiagonal components of the two-point functions of the mixing fields. These are solutions of the corresponding Kadanoff-Baym equations. For Gaussian initial conditions the latter take the form [35]

$$
\begin{aligned}
& {\left[\square_{x}+M_{i k}^{2}\right] G_{F}^{k j}(x, y)=\int_{t_{0}}^{y^{0}} d^{4} z \Pi_{F}^{i k}(x, z) G_{\rho}^{k j}(z, y)-\int_{t_{0}}^{x^{0}} d^{4} z \Pi_{\rho}^{i k}(x, z) G_{F}^{k j}(z, y),} \\
& {\left[\square_{x}+M_{i k}^{2}\right] G_{\rho}^{k j}(x, y)=\int_{x^{0}}^{y^{0}} d^{4} z \Pi_{\rho}^{i k}(x, z) G_{\rho}^{k j}(z, y),}
\end{aligned}
$$

where $M_{i j}$ are mass parameters of the renormalized Lagrangian and $\Pi_{F, \rho}^{i j}$ are renormalized self-energies. At one-loop level the self-energies are given by [34, 35]

$$
\begin{aligned}
& \Pi_{F}^{i j}(x, y)=-\frac{1}{2} H_{i j}\left[D_{F}^{2}(x, y)-\frac{1}{4} D_{\rho}^{2}(x, y)\right]-\frac{1}{2} H_{i j}^{*}\left[D_{F}^{2}(y, x)-\frac{1}{4} D_{\rho}^{2}(y, x)\right], \\
& \Pi_{\rho}^{i j}(x, y)=-\frac{1}{2} H_{i j}\left[2 D_{F}(x, y) D_{\rho}(x, y)\right]+\frac{1}{2} H_{i j}^{*}\left[2 D_{F}(y, x) D_{\rho}(y, x)\right] .
\end{aligned}
$$

Comparing (2.30) to (2.23) we conclude that in a $C$-symmetric configuration

$$
\Pi_{F(\rho)}^{i j}(x, y)=-\operatorname{Re} H_{i j} \Pi_{F(\rho)}(x, y) .
$$

In addition to the statistical and spectral propagators it is also convenient to introduce the retarded and advanced ones,

$$
\begin{aligned}
& G_{R}^{i j}(x, y) \equiv \theta\left(x^{0}-y^{0}\right) G_{\rho}^{i j}(x, y), \\
& G_{A}^{i j}(x, y) \equiv-\theta\left(y^{0}-x^{0}\right) G_{\rho}^{i j}(x, y) .
\end{aligned}
$$

The Kadanoff-Baym equations for the retarded and advanced propagators can be derived from $(2.29 \mathrm{~b})$ :

$$
\left[\square_{x}+M_{i k}^{2}\right] G_{R(A)}^{k j}(x, y)=\delta(x-y) \delta^{i j}-\int d^{4} z \Pi_{R(A)}^{i k}(x, z) G_{R(A)}^{k j}(z, y) .
$$

Explicit expressions for the retarded and advanced self-energies can be obtained from $(2.30 \mathrm{~b})$ :

$$
\Pi_{R(A)}^{i j}(x, y)=-\frac{1}{2} H_{i j}\left[2 D_{F}(x, y) D_{R(A)}(x, y)\right]-\frac{1}{2} H_{i j}^{*}\left[2 D_{F}(y, x) D_{A(R)}(y, x)\right] .
$$

Since $D_{F}^{s}(x, y)=D_{F}^{s}(y, x)$ and $D_{R}^{s}(x, y)=D_{A}^{s}(y, x)$ in a $C$-symmetric configuration we conclude that, similarly to (2.31):

$$
\Pi_{R(A)}^{i j}(x, y)=-\operatorname{Re} H_{i j} \Pi_{R(A)}(x, y),
$$

where we have introduced

$$
\Pi_{R(A)}(x, y) \equiv 2 D_{F}^{s}(x, y) D_{R(A)}^{s}(x, y) .
$$


Using the definitions of the retarded and advanced propagators (2.32), we can rewrite the Kadanoff-Baym equations (2.29) in the form

$$
\begin{aligned}
{\left[\square_{x}+M_{i k}^{2}\right] G_{F(\rho)}^{k j}(x, y)=} & -\int d^{4} z \theta\left(z_{0}-t_{0}\right) \\
& \times\left[\Pi_{F(\rho)}^{i k}(x, z) G_{A}^{k j}(z, y)+\Pi_{R}^{i k}(x, z) G_{F(\rho)}^{k j}(z, y)\right] .
\end{aligned}
$$

In thermal equilibrium all two-point functions must be translationally invariant. Wignertransforming the left- and right hand side of (2.33) we obtain,

$$
\Omega_{R(A)}^{i k}(q) G_{R(A)}^{k j}(q)=-\delta_{i j},
$$

where we have introduced

$$
\Omega_{R(A)}^{i k}(q) \equiv q^{2} \delta_{i k}-M_{i k}^{2}-\Pi_{R(A)}^{i k}(q) .
$$

To reach exact thermal equilibrium the system needs an infinite amount of time. Therefore, in order to obtain an equilibrium solution for the statistical propagator and spectral function we should send the initial time $t_{0}$ in (2.37) to minus infinity. Wignertransforming (2.37) and using the explicit form of the equilibrium solution for the retarded propagator, eq. (2.38), we obtain

$$
G_{F(\rho)}^{i j}(q)=-G_{R}^{i k}(q) \Pi_{F(\rho)}^{k l}(q) G_{A}^{l j}(q) .
$$

Using the Kubo-Martin-Schwinger (KMS) relation one can show that in thermal equilibrium the statistical propagator is proportional to the spectral function,

$$
G_{F}^{i j}(q)=\left[\frac{1}{2}+f(q u)\right] G_{\rho}^{i j}(q)
$$

where $u$ is the four-velocity of the medium and $f$ is the Bose-Einstein distribution function. Note that the four-vector $q$ in (2.41) is not constrained to be on-shell. This implies that in equilibrium the spectral shape of the statistical propagator is determined by the shape of the spectral function. Let us also note that at one-loop level (2.41) also follows from (2.31) and (2.40), which serves as a cross-check of the calculation.

A non-equilibrium solution. Using (2.33) one can show that

$$
G_{F(\rho)}^{i j}(x, y)=-\int_{t_{0}}^{\infty} d^{4} u \int_{t_{0}}^{\infty} d^{4} v G_{R}^{i k}(x, u) \Pi_{F(\rho)}^{k l}(u, v) G_{A}^{l j}(v, y),
$$

is a solution of the Kadanoff-Baym equations (2.29) for any value of $t_{0}$. For $t_{0} \rightarrow-\infty$ its Wigner-transform reverts to (2.40).

The assumption that the complex field forms a thermal bath makes the one-loop selfenergies $\Pi_{F(\rho)}$ translationally invariant. The translational invariance of the self-energies essentially renders the Kadanoff-Baym equations (2.29) linear. Therefore a sum of two solutions is also a solution. Motivated by the form of $(2.42)$ we consider [31-33, 62]

$$
\begin{aligned}
& \Delta G_{\rho}^{i j}(x, y)=0 \\
& \Delta G_{F}^{i j}(x, y)=-\int d^{3} u \int d^{3} v G_{R}^{i k}\left(x^{0}, \mathbf{x}-\mathbf{u}\right) \Delta_{F}^{k l}(\mathbf{u}-\mathbf{v}) G_{A}^{l j}\left(-y^{0}, \mathbf{v}-\mathbf{y}\right) .
\end{aligned}
$$


Substituting (2.43) into (2.29) and using (2.33) we see that it solves the Kadanoff-Baym equations, except for Dirac-deltas located on the initial time surface. In other words, (2.43) is a weak solution of (2.29). The delta-functions can be associated with external sources that (instantly) bring the system out of equilibrium at $t=0$. As can be inferred from the form of (2.43), it is not time-translationally invariant, but is space-translationally invariant. Therefore, it is convenient to perform the partial Wigner transformation,

$$
\begin{aligned}
& \Delta G_{\rho}^{i j}\left(x^{0}, y^{0}, \mathbf{q}\right)=0, \\
& \Delta G_{F}^{i j}\left(x^{0}, y^{0}, \mathbf{q}\right)=-G_{R}^{i k}\left(x^{0}, \mathbf{q}\right) \Delta_{F}^{k l}(\mathbf{q}) G_{A}^{l j}\left(-y^{0}, \mathbf{q}\right) .
\end{aligned}
$$

Physically, the sum of the solutions (2.42) and (2.43) can be interpreted as follows. The system of mixing real fields coupled to a thermal bath of the complex field begins its evolution at $t_{0}=-\infty$ in a thermal state. At $t=0$ an external source instantly brings it out of equilibrium. After that it slowly thermalises producing some asymmetry. Because the thermal bath remains in equilibrium this asymmetry would eventually be completely erased by the washout processes. However, since we neglect the latter here the asymmetry asymptotically reaches a constant value.

Of course, the washout processes are physically very important and must be taken into account in a phenomenological analysis. We would also like to stress that (2.43) is only applicable for the very peculiar instant external perturbation of the system. Nevertheless, even considering this particularly simple solution and neglecting the washout effects one can study interesting and qualitatively important features of the process of asymmetry generation in the regimes which are in principle not accessible in the other methods.

Density matrix and Boltzmann approximations. To conclude this section we will demonstrate how one can recover the Boltzmann and density matrix approximations for the source term from (2.28) and provide an interpretation for the solution (2.44) in terms of one-particle distribution functions and the density matrix respectively.

First of all we send the initial time $t_{0}$ to minus infinity and express the statistical and spectral propagators in terms of the Wightmann two-point functions. Then (2.28) takes the form

$$
\begin{aligned}
q_{S}(t)= & -i \operatorname{Im} H_{i j} \int_{-\infty}^{t} d t^{\prime} \int_{-\infty}^{t} d t^{\prime \prime} \int \frac{d^{3} q}{(2 \pi)^{3}} \frac{d^{3} p}{(2 \pi)^{3}} \frac{d^{3} k}{(2 \pi)^{3}}(2 \pi)^{3} \delta(\mathbf{q}-\mathbf{p}-\mathbf{k}) \\
& \times\left[G_{<}^{i j}\left(t^{\prime}, t^{\prime \prime}, \mathbf{q}\right) D_{>}^{s}\left(t^{\prime \prime}, t^{\prime}, \mathbf{p}\right) D_{>}^{s}\left(t^{\prime \prime}, t^{\prime}, \mathbf{k}\right)-G_{>}^{i j}\left(t^{\prime}, t^{\prime \prime}, \mathbf{q}\right) D_{<}^{s}\left(t^{\prime \prime}, t^{\prime}, \mathbf{p}\right) D_{<}^{s}\left(t^{\prime \prime}, t^{\prime}, \mathbf{k}\right)\right] .
\end{aligned}
$$

Next we introduce centre and relative time coordinates, $\tau \equiv\left(t^{\prime}+t^{\prime \prime}\right) / 2$ and $s \equiv t^{\prime}-t^{\prime \prime}$. The Jacobian determinant of this transformation is unity. Expressed in terms of the centre and relative coordinates the integral becomes

$$
\begin{aligned}
q_{S}(t)= & -i \operatorname{Im} H_{i j} \int_{-\infty}^{t} d \tau \int_{-\infty}^{+\infty} d s \int \frac{d^{3} q}{(2 \pi)^{3}} \frac{d^{3} p}{(2 \pi)^{3}} \frac{d^{3} k}{(2 \pi)^{3}}(2 \pi)^{3} \delta(\mathbf{q}-\mathbf{p}-\mathbf{k}) \\
& \times\left[G_{<}^{i j}(\tau, s, \mathbf{q}) D_{>}^{s}(\tau,-s, \mathbf{p}) D_{>}^{s}(\tau,-s, \mathbf{k})-G_{>}^{i j}(\tau, s, \mathbf{q}) D_{<}^{s}(\tau,-s, \mathbf{p}) D_{<}^{s}(\tau,-s, \mathbf{k})\right] .
\end{aligned}
$$


Introducing a Wigner-transformation with respect to the relative time,

$$
G_{\gtrless}^{i j}(\tau, s, \mathbf{q})=\int_{-\infty}^{\infty} \frac{d q_{0}}{2 \pi} e^{-i q_{0} s} G_{\gtrless}^{i j}\left(\tau, q_{0}, \mathbf{q}\right),
$$

(and a similar definition for $D_{\gtrless}$ ) we can rewrite $(2.46)$ as

$$
\begin{aligned}
q_{S}(t)= & -i \operatorname{Im} H_{i j} \int_{-\infty}^{t} d \tau \int \frac{d^{4} q}{(2 \pi)^{4}} \frac{d^{4} p}{(2 \pi)^{4}} \frac{d^{4} k}{(2 \pi)^{4}}(2 \pi)^{4} \delta(q-p-k) \\
& \times\left[G_{<}^{i j}\left(\tau, q_{0}, \mathbf{q}\right) D_{>}^{s}\left(\tau, p_{0}, \mathbf{p}\right) D_{>}^{s}\left(\tau, k_{0}, \mathbf{k}\right)-G_{>}^{i j}\left(\tau, q_{0}, \mathbf{q}\right) D_{<}^{s}\left(\tau, p_{0}, \mathbf{p}\right) D_{<}^{s}\left(\tau, k_{0}, \mathbf{k}\right)\right] .
\end{aligned}
$$

From (2.21) and (2.47) it follows that for the mixing real fields:

$$
G_{\gtrless}^{i j}\left(\tau,-q_{0},-\mathbf{q}\right)=G_{\lessgtr}^{j i}\left(\tau, q_{0}, \mathbf{q}\right) .
$$

Similarly, for the complex field in a $C$-symmetric configuration:

$$
D_{\gtrless}^{s}\left(\tau,-p_{0},-\mathbf{p}\right)=D_{\lessgtr}^{s}\left(\tau, p_{0}, \mathbf{p}\right) \text {. }
$$

Using these properties, we can reduce the integrations over positive and negative frequencies to integrations over the positive frequencies only:

$$
\begin{aligned}
q_{S}(t) & =\operatorname{Im} H_{i j} \int_{-\infty}^{t} d \tau \int \frac{\theta\left(q_{0}\right) d^{4} q}{(2 \pi)^{4}} \frac{\theta\left(p_{0}\right) d^{4} p}{(2 \pi)^{4}} \frac{\theta\left(k_{0}\right) d^{4} k}{(2 \pi)^{4}}(2 \pi)^{4} \delta(q-p-k) \\
& \times\left[\operatorname{Im} G_{<}^{i j}\left(\tau, q_{0}, \mathbf{q}\right) 2 D_{>}^{s}\left(t, p_{0}, \mathbf{p}\right) D_{>}^{s}\left(t, k_{0}, \mathbf{k}\right)-\operatorname{Im} G_{>}^{i j}\left(\tau, q_{0}, \mathbf{q}\right) 2 D_{<}^{s}\left(t, p_{0}, \mathbf{p}\right) D_{<}^{s}\left(t, k_{0}, \mathbf{k}\right)\right] .
\end{aligned}
$$

The factors of two in the squared brackets correspond to a sum of the decays into particles and antiparticles.

First we consider the Boltzmann approximation. To introduce a quasiparticle approximation for the mixing fields we note that for a hierarchical mass spectrum the diagonal (in the basis where the mass matrix is diagonal) components of the two-point functions strongly peak on the corresponding mass shells [35]. The off-diagonal components of the two-point functions are induced by the off-diagonals of the self-energy, peak at both mass shells and are small, of the order of $\Gamma / \Delta M$. Motivated by this observation, we introduce diagonal two-point functions, which are solutions of (2.38) and (2.40) with the off-diagonal components of the self-energy set to zero [35]:

$$
\begin{aligned}
\Omega_{R(A)}^{i i}(q) \mathcal{G}_{R(A)}^{i i}(q) & =-1 \\
\mathcal{G}_{F(\rho)}^{i i}(q) & =-\mathcal{G}_{R}^{i i}(q) \Pi_{F(\rho)}^{i i}(q) \mathcal{G}_{A}^{i i}(q) .
\end{aligned}
$$

The diagonal spectral function strongly peaks on the corresponding mass shell and in the limit of vanishing decay width it can be approximated by a delta-function:

$$
\mathcal{G}_{\rho}^{i i}(q)=(2 \pi) \operatorname{sign}\left(q_{0}\right) \delta\left(q^{2}-M_{i}^{2}\right)
$$


Similarly to (2.41), in equilibrium:

$$
\mathcal{G}_{F}^{i i}\left(q_{0}, \mathbf{q}\right)=\left[\frac{1}{2}+f_{i}(q u)\right] \mathcal{G}_{\rho}^{i i}\left(q_{0}, \mathbf{q}\right) .
$$

Motivated by (2.54) we use the Kadanoff-Baym ansatz for the diagonal two-point functions, i.e. assume that for small deviations from equilibrium:

$$
\mathcal{G}_{F}^{i i}\left(t, q_{0}, \mathbf{q}\right)=\left[\frac{1}{2}+f_{i}(t, q u)\right] \mathcal{G}_{\rho}^{i i}\left(q_{0}, \mathbf{q}\right)
$$

Using the definitions (2.52) we can express the full statistical and spectral propagators in terms of the diagonal ones. The exact expressions can be found in [35]. Here we will need only the leading-order approximation

$$
G_{F(\rho)}^{i j}(q) \approx \delta_{i j} \mathcal{G}_{F(\rho)}^{i j}(q)-\left(1-\delta_{i j}\right)\left[\mathcal{G}_{R}^{i i}(q) \Pi_{R}^{i j}(q) \mathcal{G}_{F(\rho)}^{j j}(q)+\mathcal{G}_{F(\rho)}^{i i}(q) \Pi_{A}^{i j}(q) \mathcal{G}_{A}^{j j}(q)\right],
$$

where no summation over the indices is implied. Substituting (2.56) into (2.51), using for the diagonal propagators the Kadanoff-Baym ansatz (2.55) together with the quasiparticle approximation (2.53), as well as similar approximations for the complex field we obtain

$$
\begin{aligned}
q_{S}(t)= & \sum_{i} \int_{-\infty}^{t} d \tau \int d \Pi_{q}^{3} d \Pi_{p}^{3} d \Pi_{k}^{3}(2 \pi)^{4} \delta(q-p-k) H_{i i} \epsilon_{i} \\
& \times\left\{f_{i}(t, \mathbf{q}) 2\left[1+f_{b}^{s}(t, \mathbf{p})\right]\left[1+f_{b}^{s}(t, \mathbf{k})\right]-\left[1+f_{i}(t, \mathbf{q})\right] 2 f_{b}^{s}(t, \mathbf{p}) f_{b}^{s}(t, \mathbf{k})\right\},
\end{aligned}
$$

where $d \Pi_{q}^{3}=d^{3} q /\left[(2 \pi)^{3} 2 \omega_{q}\right]$ is the Lorentz-invariant phase-space integration measure. The $C P$-violating parameters read [35]:

$$
\epsilon_{i}=\operatorname{Im}\left(\frac{H_{i j}}{H_{i j}^{*}}\right) \frac{\left(M_{i}^{2}-M_{j}^{2}\right)\left(M_{j} \Gamma_{j} L_{\rho}\right)}{\left(M_{i}^{2}-M_{j}^{2}\right)^{2}+\left(M_{j} \Gamma_{j} L_{\rho}\right)^{2}} \approx \epsilon_{i}^{\mathrm{vac}} L_{\rho},
$$

where $\Gamma_{j}=H_{j j} /\left(16 \pi M_{j}\right)$ is the tree-level decay width and we have neglected the momentum-dependence of the denominator to obtain the second approximate equality. Note that $\epsilon_{i}$ vanish if either $\operatorname{Im} H_{12}=0, \operatorname{Re} H_{12}=0$ or $M_{2}^{2}=M_{1}^{2}$. This reflects basic $C P$-properties of the Lagrangian which we will discuss in more details in section 3 . The function $L_{\rho}$ introduced in (4.7b) takes into account medium corrections and approaches unity at zero temperature.

Because we assume that the complex field forms a thermal bath with a constant temperature the one-particle distribution functions $f_{b}^{s}$ are time-independent. The one-particle distribution functions of the real fields can be represented as a sum of the equilibrium one and a deviation from equilibrium, $f_{i}=f_{i}^{\mathrm{eq}}+\Delta f_{i}$. In agreement with the third Sakharov condition the contribution of the equilibrium part to the right-hand side of (2.57) is identically zero. The contribution induced by the deviation from equilibrium reads

$$
\begin{aligned}
q_{S}(t)= & \sum_{i} \int_{-\infty}^{t} d \tau \int d \Pi_{q}^{3} 2 \epsilon_{i} H_{i i} \Delta f_{i}(t, \mathbf{q}) \\
& \times \int d \Pi_{p}^{3} d \Pi_{k}^{3}(2 \pi)^{4} \delta(q-p-k)\left[1+f_{b}^{s}(\mathbf{p})+f_{b}^{s}(\mathbf{k})\right] .
\end{aligned}
$$


The second line of (2.59) is nothing but a full Wigner transform of (2.23b) in the quasiparticle approximation.

To interpret (2.44) in terms of one-particle distribution functions $\Delta f_{i}$ we Wignertransform it with respect to the relative time,

$$
\Delta G_{F}^{i j}\left(\tau, q_{0}, \mathbf{q}\right)=-2 \Delta_{F}^{k l}(\mathbf{q}) \int_{-\infty}^{\infty} \frac{d p_{0}}{2 \pi} G_{R}^{i k}\left(q_{0}+p_{0}, \mathbf{q}\right) G_{A}^{l j}\left(q_{0}-p_{0}, \mathbf{q}\right) \theta(\tau) e^{-2 i p_{0} \tau}
$$

Because (2.44) vanishes for $x^{0}<0$ and $y^{0}<0$, the Wigner transform vanishes for central times $\tau<0$, as is reflected by the $\theta(\tau)$. For $j=i$ in the Boltzmann approximation we can furthermore neglecting the off-diagonal components of the propagators as well as off-diagonal components of the matrix $\Delta_{F}^{k l}$. In this case we find

$$
\begin{aligned}
\Delta G_{F}^{i i}\left(\tau, q_{0}, \mathbf{q}\right) & \approx \frac{\sin \left[2\left(q_{0}-\omega_{i}\right) \tau\right]}{q_{0}\left(q_{0}-\omega_{i}\right)} \cdot \Delta f_{i}(\tau, \mathbf{q}), \\
\Delta f_{i}(\tau, \mathbf{q}) & \equiv-\frac{\Delta_{F}^{i i}(\mathbf{q})}{2 \omega_{i}} \theta(\tau) e^{-\Gamma_{i} \tau} \equiv \Delta f_{i}(0, \mathbf{q}) \theta(\tau) e^{-\Gamma_{i} \tau} .
\end{aligned}
$$

For $\omega_{i} \tau \gg 1$ the first factor in (2.61a) strongly peaks in the vicinity of the mass shell, $q_{0} \sim \omega_{i}$, and rapidly oscillates away from the mass shell. The integration in the proximity of $q_{0}=\omega_{i}$ yields a result which oscillates around $\frac{1}{2}$ with amplitude which decreases for increasing $\tau$. In other words, effectively,

$$
\Delta G_{F}^{i i}\left(\tau, q_{0}, \mathbf{q}\right) \approx \Delta f_{i}(\tau, \mathbf{q}) G_{\rho}^{i i}\left(q_{0}, \mathbf{q}\right)
$$

for $\omega_{i} \tau \gg 1$. Comparing (2.62) to $(2.55)$ we conclude that $\Delta f_{i}(\tau, \mathbf{q})$ is a one-particle distribution function. This implies that in the used approximation $\Delta_{F}^{i i}(\mathbf{q})$ parametrizes the initial deviation of the one-particle distribution function of $\psi_{i}$ from the equilibrium one (which is determined by the temperature of the thermal bath). Substituting (2.61b) into (2.59), integrating over time and reordering the terms we obtain:

$$
q_{S}(t)=2 \sum_{i}\left(1-e^{-\Gamma_{i} t}\right) \int \frac{d q^{3}}{(2 \pi)^{3}} \frac{M_{i}}{\omega_{i}} \Delta f_{i}(0, \mathbf{q}) \epsilon_{i}\left(\omega_{i}, \mathbf{q}\right) L_{\rho}\left(\omega_{i}, \mathbf{q}\right) .
$$

Note that in the small width limit, even though $\omega_{i} \tau \gg 1$ is crucial for the applicability of the approximation, we can still assume $\Gamma \tau \ll 1$ at the lower limit of the time integration. Expression (2.63) has a simple physical interpretation. In the absence of washout processes the final asymmetry is expected to be proportional to a product of the initial deviation from equilibrium, $\Delta f_{i}(0, \mathbf{q})$, and the in-medium $C P$-violating parameter, $\epsilon_{i}$. The overall factor of two in (2.63) reflects the fact that in the toy model considered here 'lepton' number is violated by two units in each decay. The factor $L_{\rho}$ comes from the difference of the gain and loss terms.It is sometimes interpreted as a medium correction to the decay width, $\Gamma_{i}^{\text {med }}=\Gamma_{i} L_{\rho}$. However, we would like to stress that the so defined effective width does not coincide with the effective width inferred from the analysis of the spectral function. Finally, the Lorentz-invariant integration measure coincides with the one that arises in 
the calculation of the decay reaction density. For comparison with the results of the firstprinciples approach a well as those obtained in the density matrix approximation discussed below it is also useful to rewrite (2.63) in the form

$$
q_{S}(t) \approx \int \frac{d^{3} q}{(2 \pi)^{3}} \Delta_{F}(\mathbf{q}) \frac{-J}{\operatorname{det} M} \frac{1}{\left(M_{1}^{2}-M_{2}^{2}\right)^{2}} \sum_{i=1,2} \frac{\Pi_{\rho}^{2}\left(\omega_{i}, \mathbf{q}\right)}{\left(2 \omega_{i}\right)^{2}} \frac{1-e^{-\Gamma_{i} t}}{\Gamma_{i}}
$$

where $J$ is the basis-invariant measure of $C$-violation, see equation (3.5), and we have assumed that $\Delta_{F}^{k l}(\mathbf{q})=\delta^{k l} \Delta_{F}(\mathbf{q})$, see section 3 for more details.

Let us now consider the density matrix approximation. The density matrix [28] is related to the Wightmann function by [66]

$$
\rho^{i j}(t, \mathbf{q})=\int_{0}^{\infty} \frac{d q_{0}}{2 \pi} 2 q_{0} G_{<}^{i j}\left(t, q_{0}, \mathbf{q}\right) .
$$

As can be inferred from (2.65) the mass shells of the Wightmann function (see section 4 for more details) are 'summed over' in (2.65), which is well motivated for a quasidegenerate mass spectrum. Using a normalization condition for the spectral function [35] and taking furthermore into account that in the setup considered here the exact spectral function is real-valued we find

$$
\int_{0}^{\infty} \frac{d q_{0}}{2 \pi} 2 q_{0} G_{>}^{i j}\left(t, q_{0}, \mathbf{q}\right)=\rho^{i j}(t, \mathbf{q})+\delta^{i j} .
$$

Using (2.65) and (2.66) we find, approximately, from (2.51)

$$
\begin{aligned}
q_{S}(t)= & \operatorname{Im} H_{i j} \int_{-\infty}^{t} d \tau \int d \Pi_{q}^{3} d \Pi_{p}^{3} d \Pi_{k}^{3}(2 \pi)^{4} \delta(q-p-k) \\
& \times \operatorname{Im}\left\{\rho^{i j}(t, \mathbf{q}) 2\left[1+f_{b}^{s}(t, \mathbf{p})\right]\left[1+f_{b}^{s}(t, \mathbf{k})\right]-\left[\delta^{i j}+\rho^{i j}(t, \mathbf{q})\right] 2 f_{b}^{s}(t, \mathbf{p}) f_{b}^{s}(t, \mathbf{k})\right\}
\end{aligned}
$$

Similarly to the Boltzman case, because the equilibrium component of $\rho^{i j}$ does not contribute to the asymmetry, we can simplify the above expression to

$$
\begin{aligned}
q_{S}(t)= & \operatorname{Im} H_{i j} \int_{-\infty}^{t} d \tau \int d \Pi_{q}^{3} 2 \operatorname{Im} \Delta \rho^{i j}(t, \mathbf{q}) \\
& \times \int d \Pi_{p}^{3} d \Pi_{k}^{3}(2 \pi)^{4} \delta(q-p-k)\left[1+f_{b}^{s}(\mathbf{p})+f_{b}^{s}(\mathbf{k})\right] .
\end{aligned}
$$

In the small width limit the off-diagonal elements of (2.60) are well approximated (for not too small $q_{0}$ ) by

$$
\begin{aligned}
\Delta G_{F}^{i j}\left(\tau, q_{0}, \mathbf{q}\right) \approx & i \Delta_{F}(\mathbf{q}) \frac{1}{M_{i}^{2}-M_{j}^{2}} \frac{\Pi_{\rho}^{i j}(\bar{\omega}, \mathbf{q})}{(2 \bar{\omega})^{2}} \\
& \times\left[\sum_{k} \frac{\sin \left[2\left(q_{0}-\omega_{k}\right) \tau\right]}{q_{0}-\omega_{k}} e^{-\Gamma_{k} \tau}-2 i \frac{\sin \left[2\left(q_{0}-\bar{\omega}\right) \tau\right]}{q_{0}-\bar{\omega}} e^{-i\left(\omega_{i}-\omega_{j}\right) \tau} e^{-\frac{1}{2}\left(\Gamma_{i}+\Gamma_{j}\right) \tau}\right],
\end{aligned}
$$


where $\bar{\omega}=\frac{1}{2}\left(\omega_{1}+\omega_{2}\right)$ and we have again assumed that $\Delta_{F}^{k l}(\mathbf{q})=\delta^{k l} \Delta_{F}(\mathbf{q})$. The $q_{0}$ integration of the first term in the square brackets of (2.69) has been discussed above. Similarly, the integration of the last term in the square brackets in the vicinity of $q_{0} \sim$ $\bar{\omega}$ gives a term that oscillates around $e^{-i\left(\omega_{i}-\omega_{j}\right) \tau} e^{-\frac{1}{2}\left(\Gamma_{i}+\Gamma_{j}\right) \tau}$ with decreasing amplitude. Taking this into account, we obtain for the off-diagonals of the density matrix:

$$
\begin{aligned}
\Delta \rho^{i j}(\tau, \mathbf{q}) \approx & i \Delta_{F}(\mathbf{q}) \frac{\bar{\omega}}{M_{i}^{2}-M_{j}^{2}} \frac{\Pi_{\rho}^{i j}(\bar{\omega}, \mathbf{q})}{(2 \bar{\omega})^{2}} \\
& \times\left[e^{-\Gamma_{i} \tau}+e^{-\Gamma_{j} \tau}-2 i e^{-i\left(\omega_{i}-\omega_{j}\right) \tau} e^{-\frac{1}{2}\left(\Gamma_{i}+\Gamma_{j}\right) \tau}\right] .
\end{aligned}
$$

Substituting (2.70) into (2.67), using the relation (2.31) and definition (3.5) we obtain for the asymmetry

$$
\begin{aligned}
q_{S}(t) \approx & \int \frac{d^{3} q}{(2 \pi)^{3}} \Delta_{F}(\mathbf{q}) \\
& \times \frac{-J}{\operatorname{det} M} \frac{1}{\left(M_{1}^{2}-M_{2}^{2}\right)^{2}} \frac{\Pi_{\rho}^{2}(\bar{\omega}, \mathbf{q})}{(2 \bar{\omega})^{2}}\left[\sum_{i=1,2} \frac{1-e^{-\Gamma_{i} t}}{\Gamma_{i}}-2 \operatorname{Re} \frac{1-e^{-i\left(\omega_{1}-\omega_{2}\right) t} e^{-\frac{1}{2}\left(\Gamma_{1}+\Gamma_{2}\right) t}}{i\left(\omega_{1}-\omega_{2}\right)+\frac{1}{2}\left(\Gamma_{1}+\Gamma_{2}\right)}\right] .
\end{aligned}
$$

Comparing with (2.64), we conclude that the first term in the square brackets of (2.71) describes $C$-violating decays of the heavy particles, whereas the second term describes coherent $C$-violating oscillations between them which are in principle absent in the Boltzmann approximation.

\section{$3 \quad$ Fundamental symmetries and dynamics}

If the system is initially in a $C$-symmetric state then a non-zero asymmetry can be generated only dynamically. In this section we show that whether the dynamics is $C$-conserving or $C$-violating is determined by symmetries of the Lagrangian under $C$-transformation. For the system under consideration the measure of dynamical $C$-violation can be parametrized by a single flavour-basis invariant combination of the couplings and mass parameters [61]. On the other hand, even if the dynamics is $C$-conserving and the initial asymmetry is zero, a non-zero asymmetry can be generated provided that the initial conditions for the mixing fields are not $C$-symmetric. Below we discuss the conditions which ensure that the Lagrangian and the initial conditions are simultaneously invariant under $C$-transformation and show that the obtained results consistently predict zero asymmetry in this case.

Charge conjugation properties. If both, the dynamics and the initial conditions, are $C$-conserving then the final asymmetry must be zero. Let us check if (2.27) is consistent with this requirement.

The information about the dynamics is encoded in the Lagrangian. Here we work in the $\overline{\mathrm{MS}}$-scheme and therefore it is sufficient to analyse only its finite part, see [61] for more details. Under $C$-transformation the fields transform as,

$$
\begin{aligned}
C b(x) C^{-1} & =\beta \bar{b}(x), \\
C \bar{b}(x) C^{-1} & =\beta^{*} b(x), \\
C \psi_{i}(x) C^{-1} & =U_{i j} \psi_{j}(x) .
\end{aligned}
$$


where $\beta$ is a phase factor, $|\beta|=1$, and $U_{i j}$ is an orthogonal transformation. The latter can be a flavour rotation or reflection:

$$
U=\left(\begin{array}{cc}
c & -s \\
s & c
\end{array}\right) \quad \text { or } \quad U=\left(\begin{array}{cc}
c & s \\
s & -c
\end{array}\right),
$$

where we have introduced $c \equiv \cos (\alpha)$ and $s \equiv \sin (\alpha)$ to shorten the notation. $C$-transforming the Lagrangian (2.1) and using (3.1), we find that it is $C$-invariant provided that

$$
\begin{aligned}
U_{i m}^{T} M_{m n}^{2} U_{n j} & =M_{i j}^{2}, \\
\beta^{2} U_{i k}^{T} h_{k} & =h_{i}^{*} .
\end{aligned}
$$

If for a given set of couplings and mass parameters, we can find $\beta$ and $U_{i j}$ such that the conditions (3.3) are fulfilled then the Lagrangian is $C$-invariant. In general, the mass matrix has non-zero off-diagonal elements. To simplify the analysis we rotate to the basis where they vanish, i.e. where $M_{i j}^{2}=\delta_{i j} M_{i}^{2}$. If $M_{1}^{2} \neq M_{2}^{2}$ the first condition is fulfilled only for $\alpha=0, \pi$ rotations and $\alpha=0, \pi$ reflections. That is, we have to consider only four choices of $U_{i j}$. The condition (3.3b) is equivalent to the requirement that $U_{i m}^{T} H_{m n} U_{n j}=H_{i j}^{*}$. For $\alpha=0, \pi$ rotations this implies $H_{12}=H_{12}^{*}$ which holds if $\operatorname{Im} H_{12}=0$. For $\alpha=0, \pi$ reflections (3.3b) implies $H_{12}=-H_{12}^{*}$, which is fulfilled if $\operatorname{Re} H_{12}=0$. Under a flavour rotation $\operatorname{Im} H_{12}$ and $\operatorname{Re} H_{12}$ transform as [61],

$$
\begin{aligned}
& \operatorname{Im} H_{12} \rightarrow \operatorname{Im} H_{12}, \\
& \operatorname{Re} H_{12} \rightarrow\left(c^{2}-s^{2}\right) \operatorname{Re} H_{12}+c s\left(H_{22}-H_{11}\right) .
\end{aligned}
$$

Therefore, in the special case $M_{1}^{2}=M_{2}^{2}$ we can always rotate to a basis where $\operatorname{Re} H_{12}=0$. This implies that the Lagrangian (2.1) is also $C$-symmetric in this case. Summarizing the above, the dynamics is $C$-conserving if either $\operatorname{Im} H_{12}=0, \operatorname{Re} H_{12}=0$ in the basis where the mass matrix is diagonal, or the mass matrix is proportional to unity. A quantity that vanishes if any of these conditions is fulfilled is

$$
J=2 \operatorname{Im} H_{12} \operatorname{Re} H_{12} M_{1} M_{2}\left(M_{2}^{2}-M_{1}^{2}\right) .
$$

As can readily be checked, this is a special case of the form of $J$ in a general flavour basis:

$$
J=\operatorname{Im} \operatorname{tr}\left(H M^{3} H^{T} M\right) .
$$

In other words, $J$ is a basis-invariant measure of $C$-and $C P$-violation in the theory. As we showed in [61] this property is preserved under renormalization.

Let us now analyse in which case the two-point functions are also $C$-symmetric. As follows from (3.1), they transform under $C$ as

$$
G_{F(\rho)}^{i j}(x, y) \rightarrow C G_{F(\rho)}^{i j}(x, y) C^{-1}=U_{i k} G_{F(\rho)}^{c, k l}(x, y) U_{l j}^{T} .
$$

This is trivially equal to $G_{F(\rho)}^{i j}(x, y)$ for $\alpha=0, \pi$ flavour rotations. Thus, if $\operatorname{Im} H_{12}=0$, then the source term must vanish irrespective of the form of the two-point functions. On 
the other hand, for $\alpha=0, \pi$ reflections (3.7) is invariant only if the two-point functions are diagonal in the basis in which the mass matrix is diagonal. Similarly, for the special case of a mass matrix proportional to unity the two-point functions must be diagonal in the basis in which $\mathrm{Re} H_{12}=0$ to ensure that no asymmetry is generated. This implies that vanishing of the invariant (3.5) is in general insufficient to ensure zero final asymmetry. Even though the dynamics is $C$-conserving in this case, the asymmetry can be 'hidden' in the initial conditions for the two-point functions of the mixing fields.

Let us examine if, given $J=0$, the equilibrium solution (2.42) is $C$-symmetric. If $\mathrm{Re}_{12}=0$ in the basis where the mass matrix is diagonal then the self-energies are diagonal. Therefore the retarded and advanced propagators are flavour-diagonal as well, see (2.38). This in turn implies that the equilibrium spectral and statistical propagators are also flavour-diagonal, see (2.40), and are therefore $C$-symmetric. If the mass matrix is proportional to unity then, for the same reasons, in the basis where $\operatorname{Re} H_{12}=0$ the spectral and statistical propagators are diagonal and are again $C$-symmetric.

On the other hand, the non-equilibrium part of the solution, eq. (2.44), is not necessarily $C$-symmetric for $J=0$. Even though the retarded and advanced propagators are automatically diagonal if $\mathrm{Re} H_{12}=0$ in the basis where the mass matrix is diagonal, the resulting matrix $\Delta G_{F}^{i j}$ is diagonal only if $\Delta_{F}^{k l}(\mathbf{q})$ is also diagonal in this basis. If this condition is not fulfilled then the source term differs from zero even if the dynamics is $C$-conserving. Similarly, if the mass matrix is proportional to unity then the non-equilibrium part of the solution is $C$-symmetric only provided that $\Delta_{F}^{k l}(\mathbf{q})$ is diagonal in the basis where $\operatorname{Re} H_{12}=0$.

Contribution of the equilibrium solution. Since (2.42) is the equilibrium solution of (2.29), its contribution to the source term is expected to vanish even for $J \neq 0$. Let us check that this is indeed the case. First of all, sending, as discussed above, the initial time to minus infinity and using the definitions (2.32) to extend the upper integration limit to plus infinity we can rewrite $(2.25)$ in the form

$$
\begin{aligned}
S(t)= & 2 \operatorname{Im} H_{i j} \int_{-\infty}^{\infty} d t^{\prime} \int \frac{d^{3} q}{(2 \pi)^{3}} \\
& \times\left[G_{F}^{i j}\left(t-t^{\prime}, \mathbf{q}\right) \Pi_{A}\left(t^{\prime}-t, \mathbf{q}\right)+G_{R}^{i j}\left(t-t^{\prime}, \mathbf{q}\right) \Pi_{F}\left(t^{\prime}-t, \mathbf{q}\right)\right],
\end{aligned}
$$

where we have taken into account the translational invariance of the equilibrium solution. Performing a Wigner-transformation with respect to the relative time,

$$
\begin{aligned}
G_{F, R, A}^{i j}(\tau, s, \mathbf{q}) & =\int_{-\infty}^{\infty} \frac{d q_{0}}{2 \pi} e^{-i q_{0} s} G_{F, R, A}^{i j}\left(\tau, q_{0}, \mathbf{q}\right), \\
G_{\rho}^{i j}(\tau, s, \mathbf{q}) & =i \int_{-\infty}^{\infty} \frac{d q_{0}}{2 \pi} e^{-i q_{0} s} G_{\rho}^{i j}\left(\tau, q_{0}, \mathbf{q}\right),
\end{aligned}
$$

we can rewrite it in the form

$$
S(t)=2 \operatorname{Im} H_{i j} \int \frac{d^{4} q}{(2 \pi)^{4}}\left[G_{F}^{i j}\left(q_{0}, \mathbf{q}\right) \Pi_{A}\left(q_{0}, \mathbf{q}\right)+G_{R}^{i j}\left(q_{0}, \mathbf{q}\right) \Pi_{F}\left(q_{0}, \mathbf{q}\right)\right] .
$$


Substituting (2.40) into (3.10) and using (2.31) together with (2.35) we can write the contribution of the equilibrium solution to the source term as

$$
S(t)=2 \operatorname{Im} H_{i j} \int \frac{d^{4} q}{(2 \pi)^{4}} \Pi_{F}\left(q_{0}, \mathbf{q}\right) G_{R}^{i k}\left(q_{0}, \mathbf{q}\right)\left[-\Pi_{A}^{k l}\left(q_{0}, \mathbf{q}\right) G_{A}^{l j}\left(q_{0}, \mathbf{q}\right)+\delta_{k j}\right] .
$$

Using the solution for the advanced propagator (2.38) we obtain:

$$
S(t)=-2 \operatorname{Im} H_{i j} \int \frac{d^{4} q}{(2 \pi)^{4}} \Pi_{F}\left(q_{0}, \mathbf{q}\right) G_{R}^{i k}\left(q_{0}, \mathbf{q}\right)\left(q^{2} \delta_{k l}-M_{k l}^{2}\right) G_{A}^{l j}\left(q_{0}, \mathbf{q}\right) .
$$

In a $C$-symmetric medium $\Pi_{F}\left(-q_{0}, \mathbf{q}\right)=\Pi_{F}\left(q_{0}, \mathbf{q}\right)$. Furthermore, $G_{R}^{i j}\left(-q_{0}, \mathbf{q}\right)=G_{A}^{j i}\left(q_{0}, \mathbf{q}\right)$. Taking this into account we can rewrite (3.12) in the form

$$
\begin{aligned}
S(t)= & -2 \operatorname{Im} H_{i j} \int \frac{\theta\left(q_{0}\right) d^{4} q}{(2 \pi)^{4}} \Pi_{F}\left(q_{0}, \mathbf{q}\right) \\
& \times\left[G_{R}^{i k}\left(q_{0}, \mathbf{q}\right)\left(q^{2} \delta_{k l}-M_{k l}^{2}\right) G_{A}^{l j}\left(q_{0}, \mathbf{q}\right)+G_{R}^{j k}\left(q_{0}, \mathbf{q}\right)\left(q^{2} \delta_{k l}-M_{k l}^{2}\right) G_{A}^{l i}\left(q_{0}, \mathbf{q}\right)\right]=0 .
\end{aligned}
$$

Because $\operatorname{Im} H_{i j}$ is antisymmetric with respect to $i \leftrightarrow j$, whereas the integrand is symmetric, the contribution of the equilibrium solution vanishes, as expected, even for $J \neq 0$.

Contribution of the non-equilibrium solution. Next we analyse the contribution of the non-equilibrium solution (2.44). Substituting it into (2.28) we obtain

$$
\begin{aligned}
q_{S}(t) & =\operatorname{Im} H_{i j} \int_{-\infty}^{t} d t^{\prime} \int_{-\infty}^{t} d t^{\prime \prime} \int \frac{d^{3} q}{(2 \pi)^{3}} G_{R}^{i k}\left(t^{\prime}, \mathbf{q}\right) \Delta_{F}^{k l}(\mathbf{q}) G_{A}^{l j}\left(-t^{\prime \prime}, \mathbf{q}\right) \Pi_{\rho}\left(t^{\prime \prime}, t^{\prime}, \mathbf{q}\right) \\
& \equiv \int \frac{d^{3} q}{(2 \pi)^{3}} \operatorname{tr} \Delta_{F}(\mathbf{q}) \eta(\mathbf{q}),
\end{aligned}
$$

where we have factored out the dependence on the initial conditions, which are encoded in $\Delta_{F}^{k l}(\mathbf{q})$, by introducing

$$
\eta^{l k}(t, \mathbf{q}) \equiv \operatorname{Im} H_{i j} \int_{0}^{t} d t^{\prime} \int_{0}^{t} d t^{\prime \prime} G_{A}^{l j}\left(-t^{\prime \prime}, \mathbf{q}\right) \Pi_{\rho}\left(t^{\prime \prime}, t^{\prime}, \mathbf{q}\right) G_{R}^{i k}\left(t^{\prime}, \mathbf{q}\right),
$$

which contains information on the strength of $C$-violation and describes how efficiently this initial deviation from equilibrium is converted into an asymmetry. Note that because of the step functions in the definition of the retarded and advanced propagators the lower integration limits in (3.15) reduce to $t_{\min }^{\prime}=t_{\min }^{\prime \prime}=0$.

Let us now verify that (3.14) disappears if the dynamics is $C$-conserving (i.e. if $J=0$ ) and simultaneously the initial conditions are $C$-symmetric. As has been discussed above, if $J=0$ because $\operatorname{Im} H_{12}=0$, then the source term must identically vanish. A quick inspection of (3.15) shows that this is indeed the case. If $J=0$ because $\operatorname{Re} H_{12}=0$ in the basis where the mass matrix is diagonal, then the self-energy is diagonal. As follows from (2.38) the retarded and advanced propagators are also diagonal in this case. Since $\operatorname{Im} H_{i i}=0$ this in turn implies that the diagonal components of $\eta^{l k}(t, \mathbf{q})$ become zero. If the non-equilibrium solution (2.44) is $C$-symmetric, i.e. if $\Delta_{F}^{k l}(t, \mathbf{q})$ is diagonal in the same basis, then (3.14) automatically vanishes, as expected. The case of equal masses is treated analogously. 
For a particularly simple choice, $\Delta_{F}^{k l}(\mathbf{q})=\delta^{k l} \Delta_{F}(\mathbf{q})$, the solution (2.44) is automatically $C$-symmetric if $J=0$. Therefore, the final asymmetry is expected to be proportional to $J$ and to vanish for $J=0$. On the other hand, one should keep in mind that for this choice both the dynamics and the initial conditions are $C$-violating if $J \neq 0$. Substituting the chosen form of $\Delta_{F}^{k l}(\mathbf{q})$ into (3.14) we obtain:

$$
q_{S}(t)=\int \frac{d^{3} q}{(2 \pi)^{3}} \Delta_{F}(\mathbf{q}) \operatorname{tr} \eta(\mathbf{q}) .
$$

Exchanging the integration variables, $t^{\prime} \leftrightarrow t^{\prime \prime}$, using the property $\Pi_{\rho}\left(t^{\prime \prime}, t^{\prime}, \mathbf{q}\right)=-\Pi_{\rho}\left(t^{\prime}, t^{\prime \prime}, \mathbf{q}\right)$ and summing over the flavour indices we find:

$$
\operatorname{tr} \eta(t, \mathbf{q})=2 \operatorname{Im} H_{12} \int_{0}^{t} d t^{\prime} \int_{0}^{t} d t^{\prime \prime} \Pi_{\rho}\left(t^{\prime \prime}, t^{\prime}, \mathbf{q}\right) G_{R}^{1 n}\left(t^{\prime}, \mathbf{q}\right) G_{A}^{n 2}\left(-t^{\prime \prime}, \mathbf{q}\right) .
$$

Replacing the retarded and advanced propagators by their full Wigner-transforms and using furthermore that $G_{R(A)}^{i j}\left(q_{0}, \mathbf{q}\right)=G_{R(A)}^{j i}\left(q_{0}, \mathbf{q}\right)$ in a $C$-symmetric medium we can rewrite (3.17) in the form

$$
\begin{aligned}
\operatorname{tr} \eta(t, \mathbf{q})= & 8 \operatorname{Im} H_{12} \int_{0}^{t} d t^{\prime} \int_{0}^{t} d t^{\prime \prime} \Pi_{\rho}\left(t^{\prime \prime}, t^{\prime}, \mathbf{q}\right) \int_{0}^{\infty} \frac{d p_{0}}{2 \pi} \int_{0}^{\infty} \frac{d k_{0}}{2 \pi} \\
& \times \operatorname{Re}\left(\left[G_{R}^{11}\left(p_{0}, \mathbf{q}\right)-G_{R}^{22}\left(p_{0}, \mathbf{q}\right)\right] e^{-i p_{0} t^{\prime}}\right) \operatorname{Re}\left(G_{A}^{12}\left(k_{0}, \mathbf{q}\right) e^{i k_{0} t^{\prime \prime}}\right) .
\end{aligned}
$$

Being a trace this expression is flavour-basis invariant and we are free to rotate to any other basis.

Proportionality to the basis-invariant measure of CP-violation. We will denote the couplings and masses in the new basis by $\mathcal{H}$ and $\mathcal{M}$ respectively. As has been argued in the previous section, $\operatorname{Im} H_{12}$ is invariant under flavour transformations and therefore such a rotation affects only the components of the retarded and advanced propagators. To evaluate (3.18) we rotate from the basis where the mass matrix is diagonal to the basis where $\operatorname{Re} \mathcal{H}_{12}=0$. The rotation angle $\alpha$ is given by

$$
2 \alpha=\arctan \left(\frac{2 \mathrm{Re} H_{12}}{H_{11}-H_{22}}\right)
$$

In the new basis the self-energies are diagonal. Note that a flavor transformation does not 'exchange' terms between the basic Lagrangian and the counterterms and therefore, in this sense, does not alter the renormalisation prescription for the self-energies. Their diagonal components are proportional to $\mathcal{H}_{i i}$ which are related to the couplings in the initial basis by

$$
\mathcal{H}_{11 / 22}=\frac{1}{2} \operatorname{tr} H \pm \frac{1}{2} \frac{H_{11}-H_{22}}{\cos 2 \alpha} .
$$

Using (3.19) and (3.20) we can express $\cos 2 \alpha$ and $\sin 2 \alpha$ in terms of the couplings in the new and original basis:

$$
\begin{aligned}
\cos 2 \alpha & =\frac{H_{11}-H_{22}}{\mathcal{H}_{11}-\mathcal{H}_{22}}, \\
\sin 2 \alpha & =\frac{2 \operatorname{Re} H_{12}}{\mathcal{H}_{11}-\mathcal{H}_{22}} .
\end{aligned}
$$


In the new basis the matrix of mass parameters of the Lagrangian is no longer diagonal. Its components read

$$
\begin{aligned}
\mathcal{M}_{11 / 22}^{2} & =\frac{1}{2} \operatorname{tr} M^{2} \mp \frac{1}{2}\left(M_{2}^{2}-M_{1}^{2}\right) \cos 2 \alpha, \\
\mathcal{M}_{12}^{2}=\mathcal{M}_{21}^{2} & =\frac{1}{2}\left(M_{2}^{2}-M_{1}^{2}\right) \sin 2 \alpha .
\end{aligned}
$$

Using (2.38), we obtain for the components of the retarded (advanced) propagator in the new basis:

$$
G_{R(A)}=\frac{-1}{\operatorname{det} \Omega_{R(A)}}\left(\begin{array}{cc}
\Omega_{R(A)}^{22} & \mathcal{M}_{12}^{2} \\
\mathcal{M}_{21}^{2} & \Omega_{R(A)}^{11}
\end{array}\right) .
$$

As follows from (3.22b) and (3.21b), the product of $\operatorname{Im} \mathcal{H}_{12}$ and $\mathcal{M}_{12}^{2}$ is proportional to the basis-invariant measure of $C$-violation:

$$
2 \operatorname{Im} \mathcal{H}_{12} \mathcal{M}_{12}^{2}=\frac{J}{\left(\mathcal{H}_{11}-\mathcal{H}_{22}\right) \operatorname{det} M} .
$$

Using (3.24) and the explicit form of the diagonal components of the retarded propagator, eq. (3.23), we can rewrite (3.18) in the form

$$
\begin{aligned}
\operatorname{tr} \eta(t, \mathbf{q})= & \frac{4 J}{\operatorname{det} M} \int_{0}^{t} d t^{\prime} \int_{0}^{t} d t^{\prime \prime} \Pi_{\rho}\left(t^{\prime \prime}, t^{\prime}, \mathbf{q}\right) \int_{0}^{\infty} \frac{d p_{0}}{2 \pi} \int_{0}^{\infty} \frac{d k_{0}}{2 \pi} \\
& \times \operatorname{Re}\left(\frac{\Delta_{R}\left(p_{0}, \mathbf{q}\right) e^{-i p_{0} t^{\prime}}}{\operatorname{det} \Omega_{R}\left(p_{0}, \mathbf{q}\right)}\right) \operatorname{Re}\left(\frac{e^{i k_{0} t^{\prime \prime}}}{\operatorname{det} \Omega_{A}\left(k_{0}, \mathbf{q}\right)}\right)
\end{aligned}
$$

where we have introduced

$$
\Delta_{R}\left(p_{0}, \mathbf{q}\right) \equiv \frac{\left(M_{2}^{2}-M_{1}^{2}\right)\left(H_{11}-H_{22}\right)}{\left(H_{11}-H_{22}\right)^{2}+\left(2 \operatorname{Re} H_{12}\right)^{2}}+\Pi_{R}\left(p_{0}, \mathbf{q}\right)
$$

and $\Pi_{R}\left(p_{0}, \mathbf{q}\right)$ is the Wigner-transform of (2.36). We have absorbed the difference $\mathcal{H}_{11}-\mathcal{H}_{22}$ in the denominator of (3.24) into the definition of $\Delta_{R}$ and used (3.21a) and (3.22a) to express $\left(\mathcal{M}_{22}-\mathcal{M}_{11}\right) /\left(\mathcal{H}_{11}-\mathcal{H}_{22}\right)$ in terms of the couplings and mass parameters in the basis where the mass matrix is diagonal. The constant part of $\Delta_{R}$ is real-valued and, as far as its contribution is concerned, can be factored out. The integrand is then symmetric under a simultaneous transformation $p_{0} \leftrightarrow k_{0}$ and $t^{\prime} \leftrightarrow t^{\prime \prime}$. Therefore, after the integration over $p_{0}$ and $k_{0}$ the result is symmetric under $t^{\prime} \leftrightarrow t^{\prime \prime}$. On the other hand, $\Pi_{\rho}\left(t^{\prime \prime}, t^{\prime}, \mathbf{q}\right)$ is antisymmetric under $t^{\prime} \leftrightarrow t^{\prime \prime}$. Thus, the contribution of the constant part of $\Delta_{R}$ identically vanishes as an integral over the product of a symmetric and an antisymmetric function and we are left with

$$
\begin{aligned}
\operatorname{tr} \eta(t, \mathbf{q})= & \frac{4 J}{\operatorname{det} M} \int_{0}^{t} d t^{\prime} \int_{0}^{t} d t^{\prime \prime} \Pi_{\rho}\left(t^{\prime \prime}, t^{\prime}, \mathbf{q}\right) \int_{0}^{\infty} \frac{d p_{0}}{2 \pi} \int_{0}^{\infty} \frac{d k_{0}}{2 \pi} \\
& \times \operatorname{Re}\left(\frac{\Pi_{R}\left(p_{0}, \mathbf{q}\right) e^{-i p_{0} t^{\prime}}}{\operatorname{det} \Omega_{R}\left(p_{0}, \mathbf{q}\right)}\right) \operatorname{Re}\left(\frac{e^{i k_{0} t^{\prime \prime}}}{\operatorname{det} \Omega_{A}\left(k_{0}, \mathbf{q}\right)}\right)
\end{aligned}
$$


As expected, for $C$-symmetric initial conditions the source term is proportional to $J$ and vanishes in the absence of dynamical $C$-violation. This is an important cross check of the self-consistency of the used formalism.

\section{$4 \quad$ Runaway and crossing regimes}

Whereas thermal effects, by definition, do not affect the couplings and mass parameters of the Lagrangian they do influence the effective masses and widths of the in-medium quasiparticle excitations. Usually, to minimize the difference between the mass parameters and the effective masses and to ensure in this way the applicability of the quasiparticle approximation one makes use of the renormalization group equations and adjusts the renormalization scale for each value of the temperature. Effectively, this approach has also been used in our previous work [62]. However, this approach has the disadvantage that the relation between the in principle measurable zero-temperature masses and widths and the generated asymmetry is not transparent. For this reason here we adopt a different approach and fix the renormalization scale at zero temperature. Even though the resulting effective masses and widths may substantially deviate from the vacuum ones, this poses no technical problems because the Kadanoff-Baym equations do not rely on the quasiparticle picture.

Because the analysis for scalars is technically considerably easier than for fermions, here we go beyond the non-relativistic regime analysed in [62] and consider also temperatures comparable to the masses. We find that, depending on the values of the couplings and mass parameters of the Lagrangian, the effective masses may either run away from each other, or cross at some point as the temperature is increased. The identification of these two regimes is one of the novel aspects of the present work.

We also demonstrate that for very small mass differences the spectral function does not generally peak at the positions corresponding to the effective masses. This means that the approximation schemes relying on the quasiparticle picture (e.g. the Boltzmann equation) are inapplicable in this case.

Effective masses and widths. The right-hand side of (3.27) depends on the mass parameters and couplings of the Lagrangian (2.1). We will consider two benchmark points:

$$
\begin{aligned}
& \text { Set } 1: \quad h_{1}=0.5 \mu, \quad h_{2}=0.8 \mu \exp (2 i / 3), \quad M_{1}=\mu \text {, } \\
& \text { Set } 2: \quad h_{1}=0.8 \mu, \quad h_{2}=0.5 \mu \exp (2 i / 3), \quad M_{1}=\mu \text {, }
\end{aligned}
$$

where $\mu$ is the $\overline{\mathrm{MS}}$ renormalization scale. The second mass parameter, $M_{2}$, can be expressed in terms of the degeneracy parameter:

$$
R \equiv \frac{M_{2}^{2}-M_{1}^{2}}{M_{1} \Gamma_{1}+M_{2} \Gamma_{2}},
$$

where $M_{i} \Gamma_{i}=H_{i i} /(16 \pi)$ [35]. This definition is motivated by the observation made in [62] that the maximal enhancement of the asymmetry occurs for $M_{2}^{2}-M_{1}^{2}=M_{1} \Gamma_{1}+M_{2} \Gamma_{2}$, i.e. for $R \sim 1$. Large $R$ correspond to a hierarchical mass spectrum and small $R$ to a quasi-degenerate mass spectrum. 
Typically, effective masses and widths are defined as real and imaginary parts of the zeros of $\operatorname{det} \Omega_{R}$ (or det $\Omega_{A}$, they differ by complex conjugation) in the complex plane,

$$
q_{0, I}= \pm \omega_{I}-\frac{i}{2} \boldsymbol{\Gamma}_{I}, \quad \omega_{I}=\left(\mathbf{q}^{2}+\mathbf{M}_{I}^{2}\right)^{\frac{1}{2}} .
$$

Note that the effective masses and widths, $\mathbf{M}_{I}$ and $\boldsymbol{\Gamma}_{I}$, are not only temperature but also momentum-dependent because the thermal medium explicitly breaks Lorentz-invariance.

The definition of the effective masses and widths as zeros of $\operatorname{det} \Omega_{R}$ is not completely self-consistent because the retarded self-energy in

$$
\operatorname{det} \Omega_{R}=\operatorname{det}\left[q^{2}-M^{2}-\Pi_{R}\right]=q^{4}-q^{2} \operatorname{tr}\left(M^{2}+\Pi_{R}\right)+\operatorname{det}\left(M^{2}+\Pi_{R}\right),
$$

either has to be evaluated for a complex $q_{0, I}$, or the imaginary part of $q_{0, I}$ has to be neglected when evaluating $\Pi_{R}$. In one-loop approximation the explicit expression for the latter reads

$$
\Pi_{R, A, \rho}(q)=\int \frac{d^{4} k}{(2 \pi)^{4}} \frac{d^{4} p}{(2 \pi)^{4}}(2 \pi)^{4} \delta(q-p-k) 2 D_{F}^{s}(p) D_{R, A, \rho}^{s}(k) .
$$

It can be represented $[34,35]$ as $\Pi_{R, A}=\Pi_{h} \pm \frac{i}{2} \Pi_{\rho}$. In the quasiparticle approximation the Wigner-transforms of the statistical and spectral propagators of the complex field are given by $[34,35]$

$$
\begin{aligned}
D_{\rho}^{s}(p) & =(2 \pi) \operatorname{sign}\left(p_{0}\right) \delta\left(p^{2}-m^{2}\right), \\
D_{F}^{s}(p) & =[1+f(p u)] D_{\rho}^{s}(p) .
\end{aligned}
$$

Using (4.6) and assuming $m=0$ we obtain from (4.5):

$$
\begin{aligned}
\Pi_{\rho}\left(q_{0}, \mathbf{q}\right) & =\frac{1}{8 \pi} L_{\rho}\left(\frac{q_{0}}{T}, \frac{|\mathbf{q}|}{T}\right), \\
L_{\rho}\left(y_{0}, y\right) & =1+\frac{2}{y} \ln \left(\frac{1-e^{-\left(y_{0}+y\right) / 2}}{1-e^{-\left(y_{0}-y\right) / 2}}\right) .
\end{aligned}
$$

The retarded and advanced propagators can also be represented in the form $D_{R(A)}=$ $D_{h} \pm \frac{i}{2} D_{\rho}$. In the quasiparticle approximation:

$$
D_{h}^{s}(p)=-\mathcal{P} \frac{1}{p^{2}},
$$

where $\mathcal{P}$ denotes principal value. The resulting dispersive self-energy $\Pi_{h}$ is divergent and must be renormalized. Using (4.8) we find in a $C$-symmetric configuration:

$$
\begin{aligned}
& \Pi_{h}\left(q_{0}, \mathbf{q}\right)=\frac{1}{8 \pi} L_{h}\left(\frac{q_{0}}{T}, \frac{|\mathbf{q}|}{T}\right)-\frac{1}{16 \pi^{2}} \ln \frac{\left|q^{2}\right|}{\mu^{2}}, \\
& L_{h}\left(y_{0}, y\right)=\frac{1}{\pi y} \int_{0}^{\infty} d z f_{\mathrm{BE}}(z) \ln \left|\frac{(2 z+y)^{2}-y_{0}^{2}}{(2 z-y)^{2}-y_{0}^{2}}\right|,
\end{aligned}
$$


where $f_{\mathrm{BE}}$ denotes the Bose-Einstein distribution with zero chemical potential. The quasiparticle approximations (4.6) and (4.8) are valid only for the real values of their arguments and therefore (4.5) is not well defined on the complex plain.

To avoid this ambiguity here we use an alternative self-consistent definition relying on the fact that to an excellent approximation

$$
\operatorname{det} \Omega_{R}^{-1}\left(q_{0}, \mathbf{q}\right) \approx \frac{Z}{\left(q_{0}^{2}-q_{0,1}^{2}\right)\left(q_{0}^{2}-q_{0,2}^{2}\right)} .
$$

Instead of searching for zeros of $\operatorname{det} \Omega_{R}$ in the complex plane we perform a three-point fit which uniquely determines $q_{0,1}$ and $q_{0,2}$ as well as $Z$. Therefore, even though the three fit parameters are complex numbers we do not need to evaluate $\Pi_{R}$ in the complex plane. Numerically, the conventional and the alternative definition proposed here give very similar results. It is important to note that, because the determinant is invariant under flavour transformations, the fit parameters as well as the resulting effective masses and widths do not depend on the choice of the flavour basis.

The temperature dependence of the effective masses and widths for the two sets of parameters is presented in figure 2. For the first parameter set the difference of the effective masses grows monotonously with the temperature. We will refer to this case as to runaway regime. It is important to keep in mind, that $q_{0,1}$ and $q_{0,2}$ enter (4.10) symmetrically. Therefore, a transformation $q_{0,1} \leftrightarrow q_{0,2}$ would 'swap' the effective masses, $\mathbf{M}_{1} \leftrightarrow \mathbf{M}_{2}$, (as well as the widths) but leave (4.10) invariant. In figure 2 we have chosen the naming convention for $\mathbf{M}_{1}$ and $\mathbf{M}_{2}$ such, that in the limit $T \rightarrow 0$ these basis-invariant quantities approach the eigenvalues of the mass matrix, $M_{1}$ and $M_{2}$. This choice is intuitive and convenient, but is not forced by any physical principle. For the second parameter set we have also ordered the effective masses such that in the limit $T \rightarrow 0$ they approach $M_{1}$ and $M_{2}$ respectively. As the temperature grows, the difference of the effective masses decreases and at some temperature they become equal. At even higher temperatures the difference of the masses starts growing again. In principle, we are free to choose the naming convention for the masses at any temperature. In other words, we can either assume that one of the effective masses continuously grows whereas the other one continuously decreases (level crossing), or assume that the effective mass that grew below the crossing temperature, begins to decrease, whereas the mass that was increasing before the crossing temperature begins to decrease (usually referred to as avoided level crossing) as the temperature grows further. As is evident from figure 2 we have chosen the former possibility which leads to exactly the same results as the other choice. Note, that due to the momentum dependence of the masses the point in which $\mathbf{M}_{1}=\mathbf{M}_{2}$ is momentum dependent as well. Therefore there is no crossing 'point' in the strict sense but an interval in which the $\mathbf{M}_{1}(\mathbf{q})=\mathbf{M}_{2}(\mathbf{q})$ for typical momenta $\mathbf{q}$.

At this point we would like to stress once again that (4.10) is very accurate even at the point of (avoided) crossing. In other words the effective masses are well defined for any temperature and value of the degeneracy parameter.

We would also like to emphasize that the definition of effective masses and widths used here is not unique and that other definitions are possible. To give an example, one could 
Effective masses, $\mathrm{R}=5$

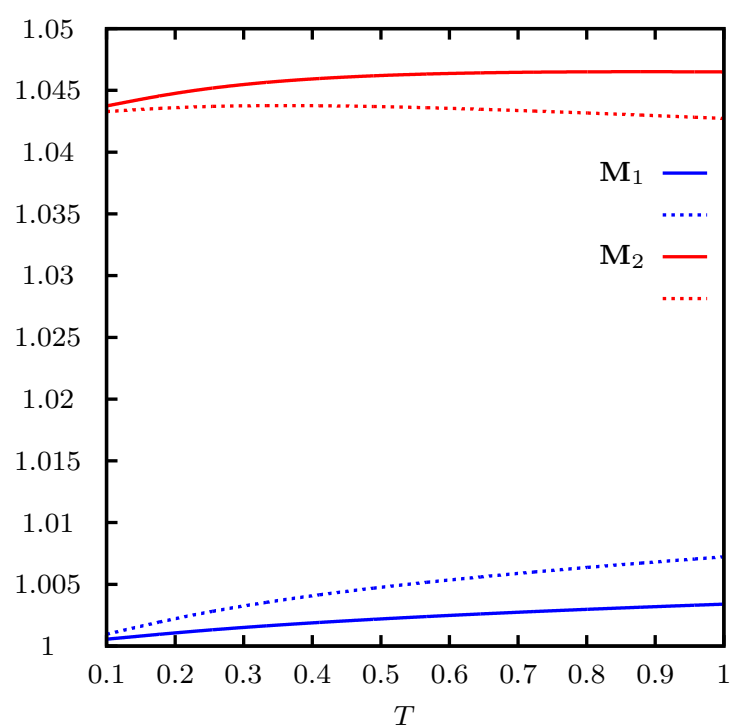

Effective masses, $\mathrm{R}=1$

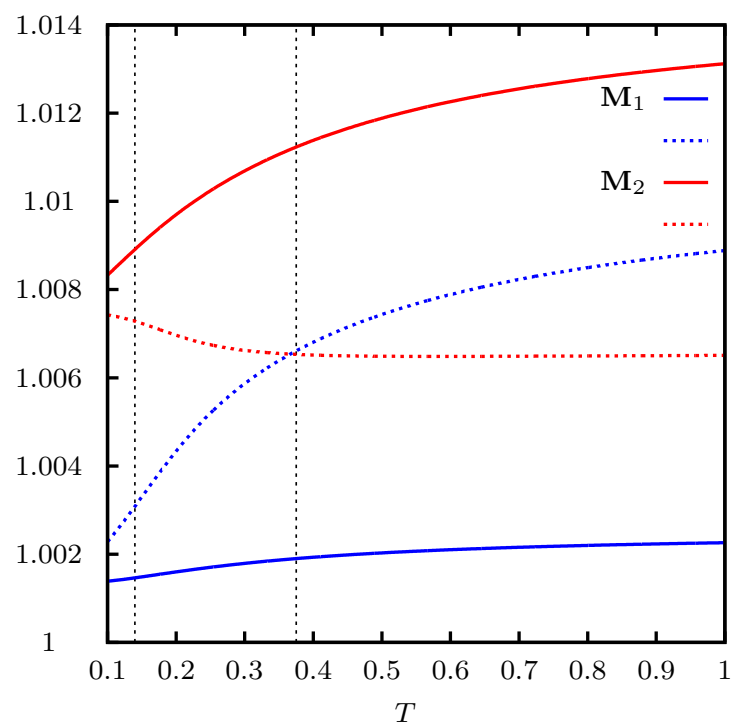

Effective widths, $\mathrm{R}=5$

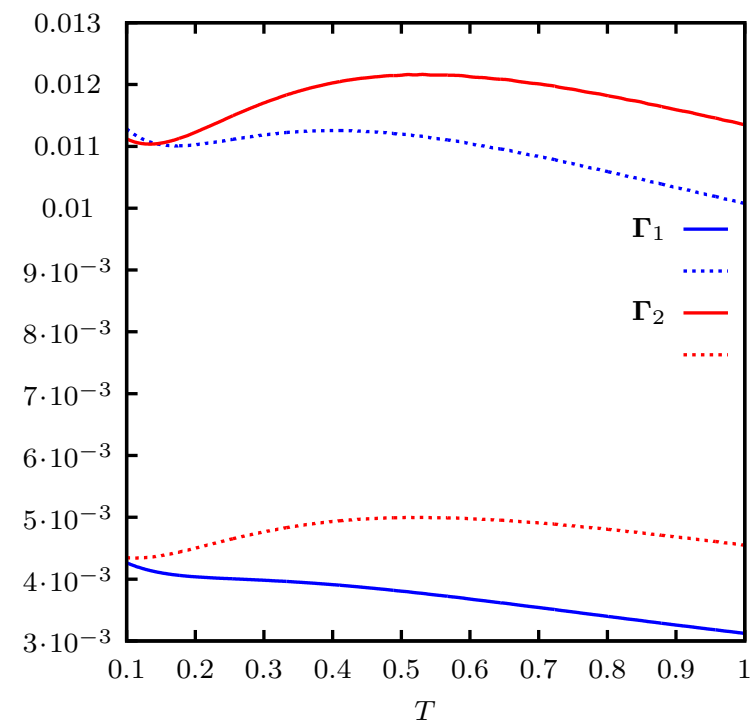

Effective widths, $\mathrm{R}=1$

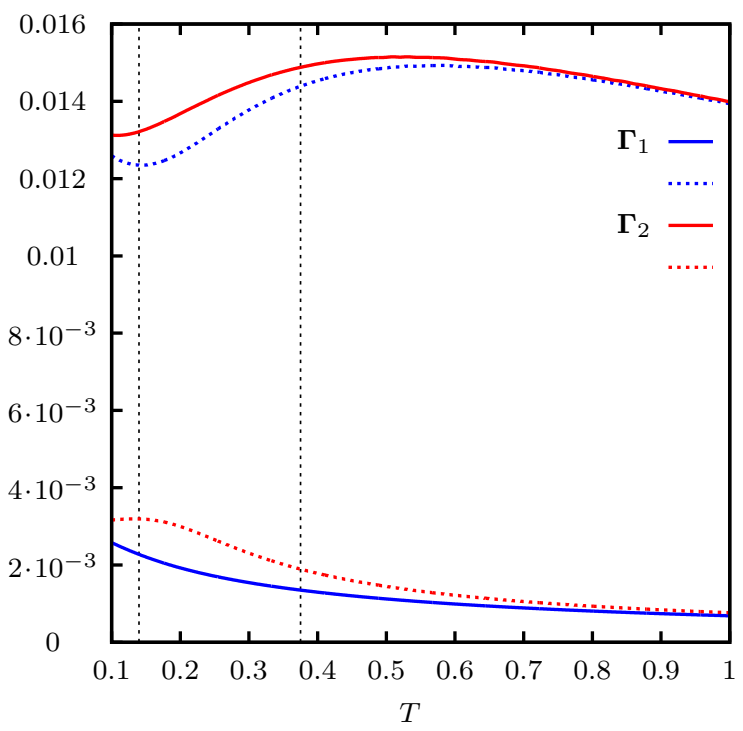

Figure 2. Temperature dependence of the effective masses and widths for $R=5$ and $R=1$. The solid lines correspond to the first parameter set, and the dotted lines to the second parameter set. For $\mathbf{q}$ we use the average thermal momentum of a boson with mass $\mu$ at each $T$. Effective masses, widths and temperature are given in units of $\mu$. The first vertical dotted line indicates the temperature for which the difference between the effective widths takes its minimal value, while the second one indicates the crossing point for which the effective masses are equal.

e.g. use $\Pi_{h}$ in (4.4) to define the effective masses. This definition would lead to a picture of avoided crossing i.e. the mass eigenvalues (for a given momentum) would never meet in a point but keep a finite minimum distance which would be reminiscent of an avoided crossing with level repulsion. The choice of the definition used in this work is motivated mainly by the fact that it leads to particularly simple and intuitive expressions for the asymmetry and other quantities. 
Equilibrium spectral function. In the setup considered here the exact spectral function coincides with the equilibrium one. The assumption that the spectral function is either diagonal in the basis where the mass matrix is diagonal or that its off-diagonal components peak at the same positions as the diagonal ones in this basis provides a starting point for various approximation schemes including the Boltzmann approximation. Components of the spectral function for two values of the degeneracy parameter and various values of temperature in the runaway and crossing regimes are presented in figure 3 . To make the comparison with figure 2 easier we have replaced the dependence of the spectral function on $q_{0}$ by a dependence on a mass parameter $M$ defined by $q_{0} \equiv\left(\mathbf{q}^{2}+M^{2}\right)^{\frac{1}{2}}$.

Given the introduced rescaling one would expect that the diagonal components of the spectral function peak at the values of $M$ equal to the values of the two effective masses, $\mathbf{M}_{1}$ and $\mathbf{M}_{2}$. Whereas this is the case for a hierarchical $(R \gtrsim 5)$ and mildly quasi-degenerate $(1 \lesssim R \lesssim 5)$ mass spectrum, we observe a rather different behaviour for a quasi-degenerate $(R \lesssim 1)$ mass spectrum, especially at high temperatures. In particular, in the runway regime at $T \sim \mu$ both diagonal components of the spectral function peak in the vicinity of $\mathbf{M}_{1}$ and do not display any non-trivial features in the vicinity of $\mathbf{M}_{2}$, see figure 3 . In the crossing regime we observe a similar behaviour.

Furthermore, the off-diagonal components differ from zero at any temperature and value of the degeneracy parameter. Note that even for a mildly hierarchical mass spectrum the peaks of the off-diagonals are not very pronounced and their positions do not exactly coincide with those of the diagonal components. For a quasi-degenerate mass spectrum the clear peak structure typical for a hierarchical mass spectrum disappears completely. In this case one of the diagonals does not have a pronounced peak and the off-diagonal components peak at only one of the mass shells. This makes the quasiparticle approximation and the use of the Boltzmann equation in this regime rather questionable.

\section{Analytical treatment of leading effects}

The dominant contribution to the asymmetry is generated by the 'difference of frequencies' terms close to the mass shell. In this section we evaluate this term analytically in the Breit-Wigner approximation. For a hierarchical mass spectrum our first-principle approach reproduces the Boltzmann approximation. We would like to stress that, in contrast to what has been contemplated in other works, in order to obtain the Boltzmann result there is no need to introduce non-zero widths of the particles forming the thermal bath. For a quasi-degenerate mass spectrum the contributions describing destructive interference between the two mass shells become important. This renders the Boltzmann approximation invalid. In particular, in the crossing regime the asymmetry computed neglecting these contributions develops a spurious peak at temperatures close to the crossing point. The negative interference terms 'remove' this peak and smoothen the dependence of the asymmetry on the temperature.

Double-time integration. To evaluate (3.27) we replace the spectral 'self-energy' $\Pi_{\rho}$ by its Wigner-transform using a relation similar to (3.9b). The double-time integration 
Spectral function in runaway regime, $\mathrm{R}=5$

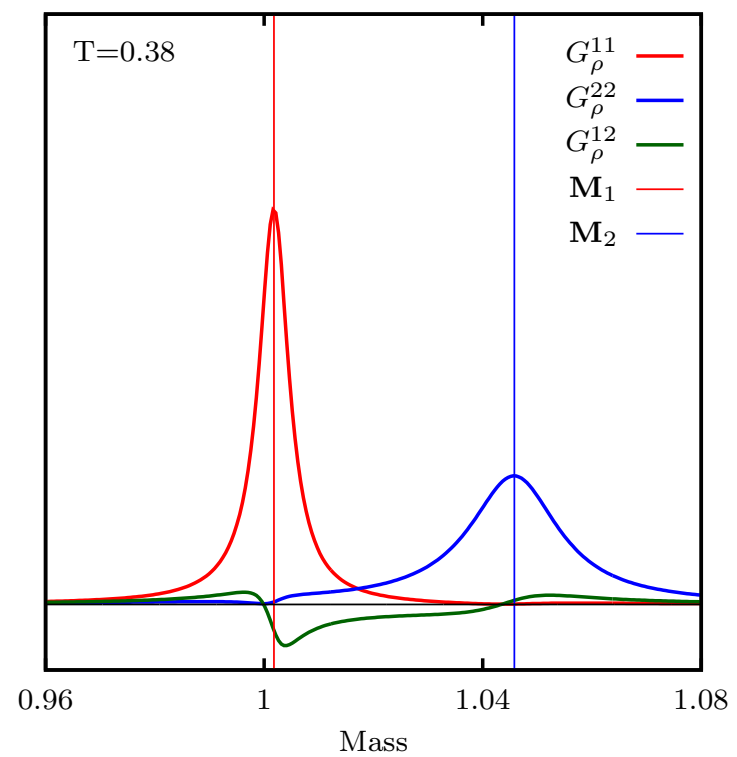

Spectral function in runaway regime, $\mathrm{R}=1$

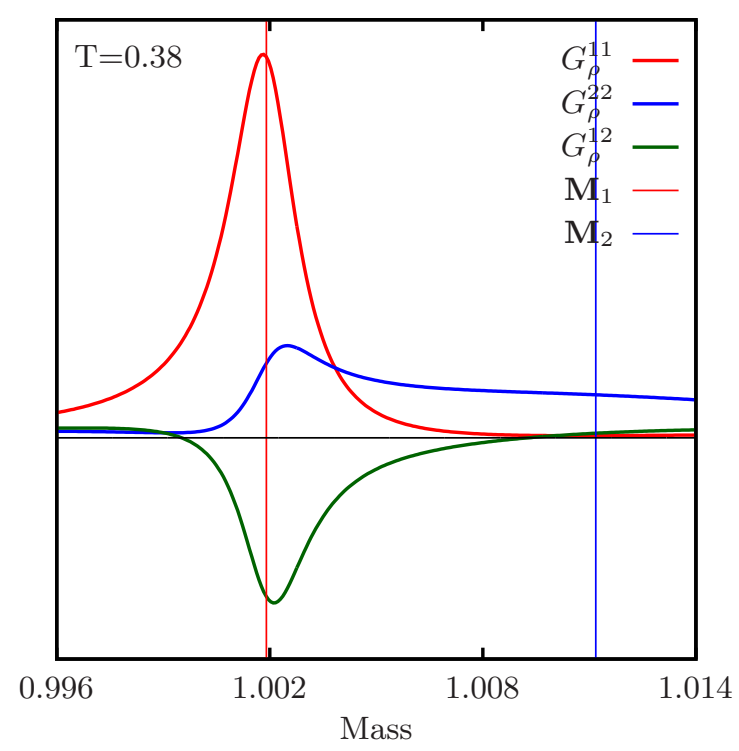

Spectral function in crossing regime, $\mathrm{R}=5$

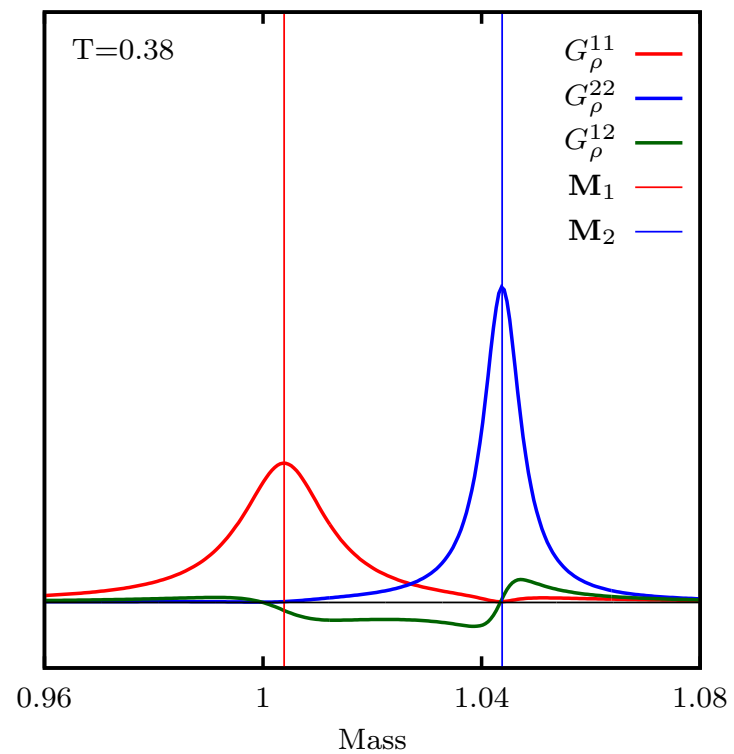

Spectral function in crossing regime, $\mathrm{R}=1$

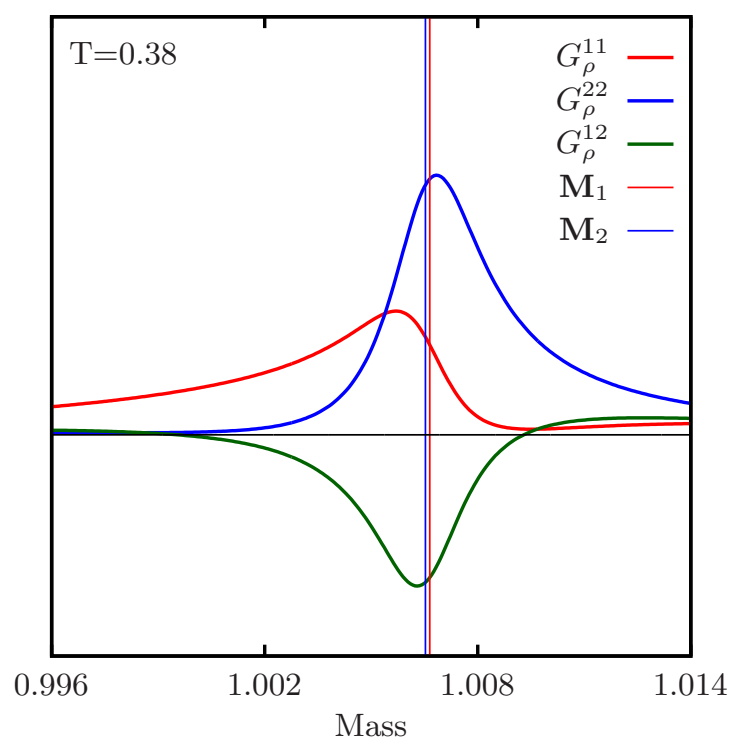

Figure 3. Components of the equilibrium spectral function for two values of the degeneracy parameter in the runaway and crossing regimes. For $\mathbf{q}$ we use average thermal momentum of a boson with mass $\mu$. To make the comparison with figure 2 easier we have replaced the dependence of the spectral function on $q_{0}$ by a dependence on a mass parameter $M$ using $q_{0} \equiv\left(\mathbf{q}^{2}+M^{2}\right)^{\frac{1}{2}}$. The solid vertical lines mark positions the effective masses $\mathbf{M}_{1}$ and $\mathbf{M}_{2}$. Effective masses and temperature are given in the units of $\mu$.

can then be performed analytically, e.g.:

$$
F\left(q_{0}, p_{0}, k_{0}, t\right) \equiv \int_{0}^{t} d t^{\prime} \int_{0}^{t} d t^{\prime \prime} e^{-i q_{0}\left(t^{\prime \prime}-t^{\prime}\right)} e^{-i p_{0} t^{\prime}} e^{i k_{0} t^{\prime \prime}}=\frac{1-e^{i\left(q_{0}-p_{0}\right) t}}{q_{0}-p_{0}} \frac{1-e^{-i\left(q_{0}-k_{0}\right) t}}{q_{0}-k_{0}} .
$$


As can readily be checked, even though the denominator of (5.1) vanishes for $q_{0}=p_{0}$ and $q_{0}=k_{0}$, the numerator simultaneously vanishes as well and the ratios are finite. Rearranging the terms, we can then rewrite (3.27) in the form

$$
\begin{aligned}
\operatorname{tr} \eta(t, \mathbf{q})=- & \frac{2 J}{\operatorname{det} M} \int_{0}^{\infty} \frac{d q_{0}}{2 \pi} \int_{0}^{\infty} \frac{d p_{0}}{2 \pi} \int_{0}^{\infty} \frac{d k_{0}}{2 \pi} \Pi_{\rho}\left(q_{0}, \mathbf{q}\right) \\
& \times \operatorname{Im}\left(\frac{\Pi_{R}\left(p_{0}, \mathbf{q}\right) F\left(q_{0}, p_{0}, k_{0}, t\right)}{\operatorname{det} \Omega_{R}\left(p_{0}, \mathbf{q}\right) \operatorname{det} \Omega_{A}\left(k_{0}, \mathbf{q}\right)}-\frac{\Pi_{R}\left(p_{0}, \mathbf{q}\right) F\left(-q_{0}, p_{0}, k_{0}, t\right)}{\operatorname{det} \Omega_{R}\left(p_{0}, \mathbf{q}\right) \operatorname{det} \Omega_{A}\left(k_{0}, \mathbf{q}\right)}\right. \\
& \left.+\frac{\Pi_{R}\left(p_{0}, \mathbf{q}\right) F\left(q_{0}, p_{0},-k_{0}, t\right)}{\operatorname{det} \Omega_{R}\left(p_{0}, \mathbf{q}\right) \operatorname{det} \Omega_{R}\left(k_{0}, \mathbf{q}\right)}-\frac{\Pi_{R}\left(p_{0}, \mathbf{q}\right) F\left(-q_{0}, p_{0},-k_{0}, t\right)}{\operatorname{det} \Omega_{R}\left(p_{0}, \mathbf{q}\right) \operatorname{det} \Omega_{R}\left(k_{0}, \mathbf{q}\right)}\right) .
\end{aligned}
$$

Because the integration in (5.2) is over positive values of $q_{0}, p_{0}$ and $k_{0}$, the dominant contribution is due to the first term where $q_{0}-p_{0}$ and $q_{0}-k_{0}$ in the denominator can vanish simultaneously. In this section we will only consider this 'difference of frequencies' contribution and neglect the other three. They are studied numerically in section 6 .

To evaluate the remaining momentum integrals we use the approximation (4.10). Introducing $x_{I} \equiv q_{0, I}^{2}-\mathbf{q}^{2}$ we can rewrite its right-hand side in the form,

$$
\frac{1}{\operatorname{det} \Omega_{R}\left(q_{0}, \mathbf{q}\right)} \approx-\frac{Z}{x_{1}-x_{2}} \frac{1}{\left(q_{0}^{2}-q_{0,1}^{2}\right)}+\frac{Z}{x_{1}-x_{2}} \frac{1}{\left(q_{0}^{2}-q_{0,2}^{2}\right)} .
$$

Similarly,

$$
\frac{1}{\operatorname{det} \Omega_{A}\left(q_{0}, \mathbf{q}\right)} \approx-\frac{Z^{*}}{x_{1}^{*}-x_{2}^{*}} \frac{1}{\left(q_{0}^{2}-q_{0,1}^{2}\right)^{*}}-\frac{Z^{*}}{x_{1}^{*}-x_{2}^{*}} \frac{1}{\left(q_{0}^{2}-q_{0,2}^{2}\right)^{*}} .
$$

Substituting these expressions, we find for the first term of (5.2):

$$
\begin{aligned}
\operatorname{tr} \eta(t, \mathbf{q})= & -\frac{2 J}{\operatorname{det} M} \frac{|Z|^{2}}{\left|x_{1}-x_{2}\right|^{2}} \int_{0}^{\infty} \frac{d q_{0}}{2 \pi} \Pi_{\rho}\left(q_{0}, \mathbf{q}\right) \\
& \times \operatorname{Im}\left[\sum_{I=1,2} \int_{0}^{\infty} \frac{d p_{0}}{2 \pi} \frac{\Pi_{R}\left(p_{0}, \mathbf{q}\right)}{p_{0}^{2}-q_{0, I}^{2}} \frac{1-e^{i\left(q_{0}-p_{0}\right) t}}{q_{0}-p_{0}} \int_{0}^{\infty} \frac{d k_{0}}{2 \pi} \frac{1}{\left(k_{0}^{2}-q_{0, I}^{2}\right)^{*}} \frac{1-e^{-i\left(q_{0}-k_{0}\right) t}}{q_{0}-k_{0}}\right. \\
& \left.-\sum_{I=1,2}^{I \neq J} \int_{0}^{\infty} \frac{d p_{0}}{2 \pi} \frac{\Pi_{R}\left(p_{0}, \mathbf{q}\right)}{p_{0}^{2}-q_{0, I}^{2}} \frac{1-e^{i\left(q_{0}-p_{0}\right) t}}{q_{0}-p_{0}} \int_{0}^{\infty} \frac{d k_{0}}{2 \pi} \frac{1}{\left(k_{0}^{2}-q_{0, J}^{2}\right)^{*}} \frac{1-e^{-i\left(q_{0}-k_{0}\right) t}}{q_{0}-k_{0}}\right] .
\end{aligned}
$$

Note that the two terms in square brackets have opposite sign, i.e. there is a destructive interference. This effect can be traced back to equations (5.3) and (5.4). In the limit $q_{0,2} \rightarrow q_{0,1}$ the difference $x_{1}-x_{2}$ in the denominator of (5.5) vanishes. However, because of the destructive interference, the numerator simultaneously vanishes as well and the ratio remains finite. This conclusion is consistent with the results of [62].

Hierarchical mass spectrum. For a hierarchical mass spectrum $q_{0,1}$ and $q_{0,2}$ are well separated and the contribution of the interference term is negligible. Therefore, it is sufficient to consider only the first term of (5.5). Because the integrands are strongly peaked 
in the vicinity of $q_{0, I}$, we can approximate $\Pi_{R}\left(p_{0}, \mathbf{q}\right)$ by $\Pi_{R}\left(\omega_{I}, \mathbf{q}\right)$. The $p_{0}$ and $k_{0}$ integrals are then complex conjugates of each other and their product is real valued. Since $\operatorname{Im} \Pi_{R}\left(\omega_{I}, \mathbf{q}\right)=\frac{1}{2} \Pi_{\rho}\left(\omega_{I}, \mathbf{q}\right)$ this results in

$$
\begin{aligned}
\operatorname{tr} \eta(t, \mathbf{q}) \approx & -\frac{J}{\operatorname{det} M} \frac{|Z|^{2}}{\left|x_{1}-x_{2}\right|^{2}} \sum_{I=1,2} \Pi_{\rho}\left(\omega_{I}, \mathbf{q}\right) \int_{0}^{\infty} \frac{d q_{0}}{2 \pi} \Pi_{\rho}\left(q_{0}, \mathbf{q}\right) \\
& \times \int_{0}^{\infty} \frac{d p_{0}}{2 \pi} \frac{1}{p_{0}^{2}-q_{0, I}^{2}} \frac{1-e^{i\left(q_{0}-p_{0}\right) t}}{q_{0}-p_{0}} \int_{0}^{\infty} \frac{d k_{0}}{2 \pi} \frac{1}{\left(k_{0}^{2}-q_{0, I}^{2}\right)^{*}} \frac{1-e^{-i\left(q_{0}-k_{0}\right) t}}{q_{0}-k_{0}} .
\end{aligned}
$$

The integrations over $p_{0}$ and $k_{0}$ give approximately:

$$
\begin{gathered}
\int_{0}^{\infty} \frac{d p_{0}}{2 \pi} \frac{1}{\left(p_{0}^{2}-q_{0, I}^{2}\right)} \frac{1-e^{i\left(q_{0}-p_{0}\right) t}}{q_{0}-p_{0}} \approx-\frac{i}{2 q_{0, I}} \frac{1-e^{i\left(q_{0}-q_{0, I}\right) t}}{q_{0}-q_{0, I}}, \\
\int_{0}^{\infty} \frac{d k_{0}}{2 \pi} \frac{1}{\left(k_{0}^{2}-q_{0, I}^{2}\right)^{*}} \frac{1-e^{-i\left(q_{0}-p_{0}\right) t}}{q_{0}-k_{0}} \approx \frac{i}{2 q_{I, 0}^{*}} \frac{1-e^{-i\left(q_{0}-q_{I, 0}^{*}\right) t}}{q_{0}-q_{I, 0}^{*}} .
\end{gathered}
$$

These expressions are valid in the vicinity of $q_{0} \approx \omega_{I}$ and result in

$$
\begin{aligned}
\operatorname{tr} \eta(t, \mathbf{q}) \approx & -\frac{J}{\operatorname{det} M} \frac{|Z|^{2}}{\left|x_{1}-x_{2}\right|^{2}} \sum_{I=1,2} \Pi_{\rho}\left(\omega_{I}, \mathbf{q}\right) \int_{0}^{\infty} \frac{d q_{0}}{2 \pi} \Pi_{\rho}\left(q_{0}, \mathbf{q}\right) \\
& \times \frac{1}{\left(2 \omega_{I}\right)^{2}} \frac{\left|1-e^{i\left(q_{0}-\omega_{I}\right) t} e^{-\frac{1}{2} \boldsymbol{\Gamma}_{I} t}\right|^{2}}{\left(q_{0}-\omega_{I}\right)^{2}+\left(\frac{1}{2} \boldsymbol{\Gamma}_{I}\right)^{2}}
\end{aligned}
$$

Using

$$
\lim _{\epsilon \rightarrow 0} \frac{2 \epsilon}{\omega^{2}+\epsilon^{2}}=2 \pi \delta(\omega)
$$

we can perform the integration over $q_{0}$ and obtain

$$
\operatorname{tr} \eta(t, \mathbf{q}) \approx-\frac{J}{\operatorname{det} M} \frac{|Z|^{2}}{\left|x_{1}-x_{2}\right|^{2}} \sum_{I=1,2} \frac{\Pi_{\rho}^{2}\left(\omega_{I}, \mathbf{q}\right)}{\left(2 \omega_{I}\right)^{2}} \frac{\left(1-e^{-\frac{1}{2} \boldsymbol{\Gamma}_{I} t}\right)^{2}}{\boldsymbol{\Gamma}_{I}} .
$$

The obtained time dependence, $\left(1-e^{-\frac{1}{2} \boldsymbol{\Gamma}_{I} t}\right)^{2}$, is consistent with the result of [33]. However, numerical analysis shows that this approximation is rather crude. The oscillating exponent $e^{i\left(q_{0}-\omega_{I}\right) t}$ substantially changes the shape of the peak in the vicinity of $q_{0} \approx \omega_{I}$ and renders this approximation inaccurate. On the other hand, a very accurate approximation is provided by

$$
\operatorname{tr} \eta(t, \mathbf{q}) \approx-\frac{J}{\operatorname{det} M} \frac{|Z|^{2}}{\left|x_{1}-x_{2}\right|^{2}} \sum_{I=1,2} \frac{\Pi_{\rho}^{2}\left(\omega_{I}, \mathbf{q}\right)}{\left(2 \omega_{I}\right)^{2}} \frac{1-e^{-\boldsymbol{\Gamma}_{I} t}}{\boldsymbol{\Gamma}_{I}},
$$

compare with (2.64). In other words, by taking into account the oscillating exponent we recover the time dependence expected in the Boltzmann approximation, see [33, 62], without introducing an effective width of the particles forming the thermal bath. Note that

$$
\frac{1}{\left|x_{1}-x_{2}\right|^{2}} \approx \frac{1}{\left(\mathbf{M}_{1}^{2}-\mathbf{M}_{2}^{2}\right)^{2}+\left(\omega_{1} \boldsymbol{\Gamma}_{1}-\omega_{2} \boldsymbol{\Gamma}_{2}\right)^{2}},
$$


i.e. we recover the usual resonant enhancement [52, 62]. Furthermore, combining (5.12) with the definition $J$, see (3.5), we find that the product of the first two terms in (5.11) is equal to,

$$
-\frac{J}{\operatorname{det} M} \frac{1}{\left|x_{1}-x_{2}\right|^{2}}=\frac{\operatorname{Im}\left(H_{12}^{2}\right)\left(M_{2}^{2}-M_{1}^{2}\right)}{\left(\mathbf{M}_{1}^{2}-\mathbf{M}_{2}^{2}\right)^{2}+\left(\omega_{1} \boldsymbol{\Gamma}_{1}-\omega_{2} \boldsymbol{\Gamma}_{2}\right)^{2}},
$$

which strongly resembles equation (137) of [62] found there empirically using numerical analysis. The peculiarity of this expression is that its numerator contains the mass parameters of the Lagrangian, whereas the denominator contains effective masses and widths.

Let us finally compare (5.11) with (2.63). For a strongly hierarchical mass spectrum the deviation of the thermal masses and widths from the vacuum ones is negligible. Furthermore, the $M_{i} \Gamma_{i}$ terms are small compared to $M_{i}^{2}-M_{j}^{2}$. Therefore (5.13) is approximately equal to $(16 \pi)^{2} \epsilon_{i}^{\mathrm{vac}} M_{i} \Gamma_{i}$. Taking into account that $\Pi_{\rho}=L_{\rho} /(8 \pi)$ and using $(2.58)$ we obtain,

$$
\operatorname{tr} \eta(t, \mathbf{q})=2 \sum_{i}\left(1-e^{-\Gamma_{i} t}\right) \frac{1}{2 \omega_{i}} \frac{M_{i}}{\omega_{i}} \epsilon_{i}\left(\omega_{i}, \mathbf{q}\right) L_{\rho}\left(\omega_{i}, \mathbf{q}\right)
$$

In other words, $\eta$ can be viewed as a weighted average of the in-medium $C P$-violating parameter with some kinematical and thermal functions. Substituting (5.14) into (3.16) and recalling the relation between $\Delta_{F}(\mathbf{q})$ and $\Delta f(0, \mathbf{q})$ we see that the resulting expression for $q_{S}(t)$ is identical to $(2.63)$.

Quasidegenerate mass spectrum. For a quasi-degenerate mass spectrum $q_{0,1}$ and $q_{0,2}$ are very close and we need to take into account also the contributions of the $J \neq I$ term in (5.5). Since away of $p_{0}^{2}=\mathbf{q}^{2}$ region $\Pi_{R}\left(p_{0}, \mathbf{q}\right)$ is a slowly varying function of $p_{0}$ we can approximate it by $\Pi_{R}(\bar{\omega}, \mathbf{q})$, where $\bar{\omega} \equiv \frac{1}{2}\left(\omega_{1}+\omega_{2}\right)$. In this approximation the products ( $J=I$ contributions) and the sum of the products $(J \neq I$ contributions $)$ of the momentum integrals in (5.5) are again real valued and we obtain

$$
\begin{aligned}
\operatorname{tr} \eta(t, \mathbf{q})= & -\frac{J}{\operatorname{det} M} \frac{|Z|^{2}}{\left|x_{1}-x_{2}\right|^{2}} \Pi_{\rho}(\bar{\omega}, \mathbf{q}) \int_{0}^{\infty} \frac{d q_{0}}{2 \pi} \Pi_{\rho}\left(q_{0}, \mathbf{q}\right) \\
& \times\left[\sum_{I=1,2} \int_{0}^{\infty} \frac{d p_{0}}{2 \pi} \frac{1}{p_{0}^{2}-q_{0, I}^{2}} \frac{1-e^{i\left(q_{0}-p_{0}\right) t}}{q_{0}-p_{0}} \int_{0}^{\infty} \frac{d k_{0}}{2 \pi} \frac{1}{\left(k_{0}^{2}-q_{0, I}^{2}\right)^{*}} \frac{1-e^{-i\left(q_{0}-k_{0}\right) t}}{q_{0}-k_{0}}\right. \\
& \left.-2 \operatorname{Re} \int_{0}^{\infty} \frac{d p_{0}}{2 \pi} \frac{1}{p_{0}^{2}-q_{0,1}^{2}} \frac{1-e^{i\left(q_{0}-p_{0}\right) t}}{q_{0}-p_{0}} \int_{0}^{\infty} \frac{d k_{0}}{2 \pi} \frac{1}{\left(k_{0}^{2}-q_{0,2}^{2}\right)^{*}} \frac{1-e^{-i\left(q_{0}-k_{0}\right) t}}{q_{0}-k_{0}}\right] .
\end{aligned}
$$

Using approximations (5.7) and integrating over $q_{0}$ we arrive at

$$
\begin{aligned}
\operatorname{tr} \eta(t, \mathbf{q}) \approx & -\frac{J}{\operatorname{det} M} \frac{|Z|^{2}}{\left|x_{1}-x_{2}\right|^{2}} \frac{\Pi_{\rho}^{2}(\bar{\omega}, \mathbf{q})}{(2 \bar{\omega})^{2}} \\
& \times\left[\sum_{I=1,2} \frac{1-e^{-\boldsymbol{\Gamma}_{I} t}}{\boldsymbol{\Gamma}_{I}}-2 \operatorname{Re} \frac{1-e^{-i\left(\omega_{1}-\omega_{2}\right) t} e^{-\frac{1}{2}\left(\boldsymbol{\Gamma}_{1}+\boldsymbol{\Gamma}_{2}\right) t}}{i\left(\omega_{1}-\omega_{2}\right)+\frac{1}{2}\left(\boldsymbol{\Gamma}_{1}+\boldsymbol{\Gamma}_{2}\right)}\right],
\end{aligned}
$$



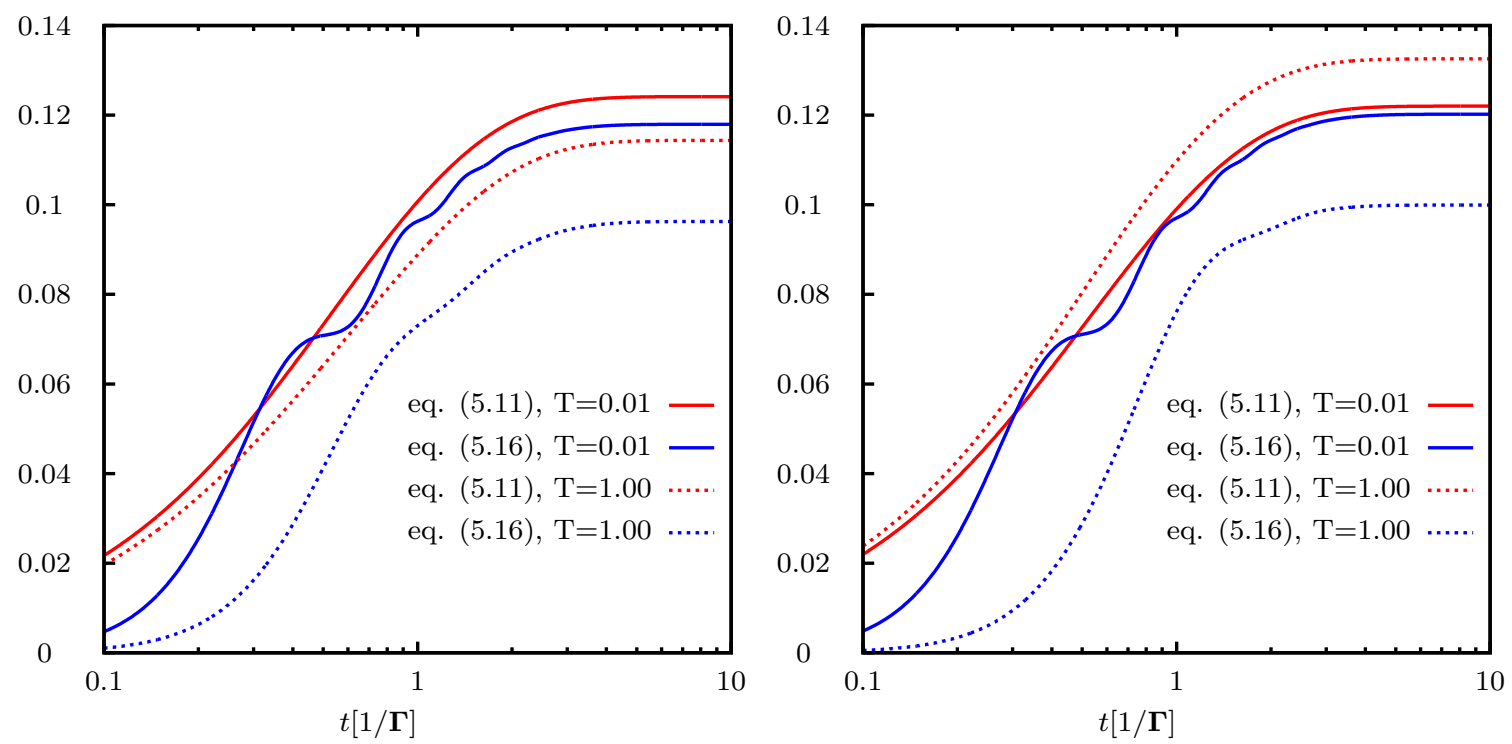

Figure 4. Time dependence of the two approximate expressions for $\operatorname{tr} \eta$ (in units of $1 / \mu$ ) in the runaway and crossing regimes for $R=5$ and two values of the temperature, $T=0.01$ and $T=1$ (in units of $\mu$ ). Time is given in units of $1 / \boldsymbol{\Gamma} \equiv 1 / \boldsymbol{\Gamma}_{1}+1 / \boldsymbol{\Gamma}_{2}$.

which is analogous to the result of [62], compare also with (2.71). As has been discussed above, if $\mathbf{M}_{2} \rightarrow \mathbf{M}_{1}$ and $\boldsymbol{\Gamma}_{2} \rightarrow \boldsymbol{\Gamma}_{1}$ simultaneously, then both the numerator and the difference $x_{1}-x_{2}$ in the denominator of (5.16) vanish simultaneously, but their ratio remains finite.

For illustration we present the time-dependence of the two expressions for $\operatorname{tr} \eta$, eq. (5.11) and eq. (5.16) in figure 4 , for $R=5$. For this value of the degeneracy parameter both the hierarchical and quasi-degenerate approximations are expected to be reasonably good. At low temperatures the two approximations give similar results. On the other hand at high temperatures they yield values for the final asymmetry which differ by roughly $40 \%$.

Asymptotic behaviour. For $t \rightarrow \infty$ the hierarchical and quasi-degenerate approximations, eq. (5.11) and eq. (5.16), simplify to

$$
\begin{aligned}
\operatorname{tr} \eta(\infty, \mathbf{q})= & -\frac{J}{\operatorname{det} M} \frac{|Z|^{2}}{\left|x_{1}-x_{2}\right|^{2}} \sum_{I=1,2} \frac{\Pi_{\rho}^{2}\left(\omega_{I}, \mathbf{q}\right)}{\left(2 \omega_{I}\right)^{2}} \frac{1}{\boldsymbol{\Gamma}_{I}}, \\
\operatorname{tr} \eta(\infty, \mathbf{q})= & -\frac{J}{\operatorname{det} M} \frac{|Z|^{2}}{\left|x_{1}-x_{2}\right|^{2}} \frac{\Pi_{\rho}^{2}\left(\bar{\omega}_{\mathbf{q}}, \mathbf{q}\right)}{\left(2 \bar{\omega}_{\mathbf{q}}\right)^{2}} \\
& \times\left[\sum_{I=1,2} \frac{1}{\Gamma_{I}}-2 \operatorname{Re} \frac{1}{i\left(\omega_{1}-\omega_{2}\right)+\frac{1}{2}\left(\boldsymbol{\Gamma}_{1}+\boldsymbol{\Gamma}_{2}\right)}\right] .
\end{aligned}
$$

We can try to improve the estimate of the asymptotic value of the asymmetry. To this end we note that for $t \rightarrow \infty$ the contributions of the oscillating terms in (5.6) and (5.15) 
vanish. The integrations over $p_{0}$ and $k_{0}$ can then be performed analytically:

$$
\begin{aligned}
& \int_{0}^{\infty} \frac{d p_{0}}{2 \pi} \frac{1}{p_{0}^{2}-q_{0, I}^{2}} \frac{1-e^{i\left(q_{0}-p_{0}\right) t}}{q_{0}-p_{0}} \rightarrow \lim _{\epsilon \rightarrow 0} \int_{0}^{\infty} \frac{d p_{0}}{2 \pi} \frac{1}{\left(p_{0}^{2}-q_{0, I}^{2}\right)} \frac{1}{q_{0}-p_{0}+i \epsilon} \\
& =\frac{1}{2 \pi}\left(\frac{\ln \left(q_{0}\right)-i \pi}{\left(q_{0}^{2}-q_{0, I}^{2}\right)}+\frac{1}{2 q_{0, I}} \frac{\ln \left(q_{0, I}\right)}{q_{0}+q_{0, I}}-\frac{1}{2 q_{0, I}} \frac{\ln \left(-q_{0, I}\right)}{q_{0}-q_{0, I}}\right), \\
& \int_{0}^{\infty} \frac{d k_{0}}{2 \pi} \frac{1}{\left(k_{0}^{2}-q_{0, I}^{2}\right)^{*}} \frac{1-e^{-i\left(q_{0}-k_{0}\right) t}}{q_{0}-k_{0}} \rightarrow \lim _{\epsilon \rightarrow 0} \int_{0}^{\infty} \frac{d k_{0}}{2 \pi} \frac{1}{\left(k_{0}^{2}-q_{0, I}^{2}\right)^{*}} \frac{1}{q_{0}-k_{0}-i \epsilon} \\
& =\frac{1}{2 \pi}\left(\frac{\ln \left(q_{0}\right)+i \pi}{\left(q_{0}^{2}-q_{0, I}^{2}\right)^{*}}+\frac{1}{2 q_{0, I}^{*}} \frac{\ln \left(q_{0, I}^{*}\right)}{q_{0}+q_{0, I}^{*}}-\frac{1}{2 q_{0, I}^{*}} \frac{\ln \left(-q_{0, I}^{*}\right)}{q_{0}-q_{0, I}^{*}}\right) .
\end{aligned}
$$

A numerical comparison of the $q_{0}$ integrands computed using (5.7) and (5.18) shows that in the relevant range of $q_{0}$ the difference between the two is completely negligible even at the crossing point. In other words, (5.17a) provides a very accurate estimate of the asymmetry for a strongly hierarchical mass spectrum, whereas $(5.17 \mathrm{~b})$ provides a very accurate estimate of the asymmetry for a quasi-degenerate mass spectrum. Their temperature dependence in the runaway and crossing regimes for $R=1$ is presented in figure 6 . In the crossing regime the hierarchical approximation for $\operatorname{tr} \eta$ develops a spurious peak. Contrary to what one would naively expect the peak is located at a temperature somewhat lower than the crossing temperature. This can be traced back to the second term in the denominator of (5.13). The effective widths also depend on the temperature, see figure 2. For $R=1$ the difference of the effective widths reaches minimum around $T \sim 0.14$, whereas the difference of the effective masses reaches minimum around $T \sim 0.38$. As a result $\operatorname{tr} \eta$ computed in the Boltzmann approximation peaks at a temperature between these two. The contribution of the negative interference terms ensures that the quasi-degenerate approximation for $\operatorname{tr} \eta$ remains smooth for all values of $T$ and does not develop a peak.

For intermediate values of $R$ neither (5.17a) nor (5.17b) is applicable. To obtain an analytical expression valid for any $R$ we note that in the $p_{0}$ integrand in (5.5) it is sufficient to approximate $\Pi_{R}\left(p_{0}, \mathbf{q}\right)$ by $\Pi_{R}\left(\omega_{I}, \mathbf{q}\right)$. Furthermore, in the relevant $q_{0}$ range $\Pi_{\rho}\left(q_{0}, \mathbf{q}\right)$ is a smooth function of $q_{0}$ and is well approximated by its linear expansion. The integration then results in

$$
\begin{aligned}
\operatorname{tr} \eta(\infty, \mathbf{q})= & -\frac{J}{\operatorname{det} M} \frac{|Z|^{2}}{\left|x_{1}-x_{2}\right|^{2}}\left[\sum_{I=1,2} \frac{\Pi_{\rho}^{2}\left(\omega_{I}, \mathbf{q}\right)}{\left(2 \omega_{I}\right)^{2}} \frac{1}{\boldsymbol{\Gamma}_{I}}\right. \\
& -\frac{1}{2} \frac{\Pi_{\rho}\left(\omega_{1}, \mathbf{q}\right)+\Pi_{\rho}\left(\omega_{2}, \mathbf{q}\right)}{\left(2 \omega_{1}\right)\left(2 \omega_{2}\right)} \operatorname{Re} \frac{\Pi_{\rho}\left(\omega_{1}, \mathbf{q}\right)(1-i \delta)+\Pi_{\rho}\left(\omega_{2}, \mathbf{q}\right)(1+i \delta)}{i\left(\omega_{1}-\omega_{2}\right)+\frac{1}{2}\left(\boldsymbol{\Gamma}_{1}+\boldsymbol{\Gamma}_{2}\right)} \\
& \left.-\frac{1}{4} \frac{\Pi_{h}\left(\omega_{1}, \mathbf{q}\right)-\Pi_{h}\left(\omega_{2}, \mathbf{q}\right)}{\left(2 \omega_{1}\right)\left(2 \omega_{2}\right)} \operatorname{Im} \frac{\Pi_{\rho}\left(\omega_{1}, \mathbf{q}\right)(1-i \delta)+\Pi_{\rho}\left(\omega_{2}, \mathbf{q}\right)(1+i \delta)}{i\left(\omega_{1}-\omega_{2}\right)+\frac{1}{2}\left(\boldsymbol{\Gamma}_{1}+\boldsymbol{\Gamma}_{2}\right)}\right],
\end{aligned}
$$

where $\delta \equiv \frac{1}{2}\left(\boldsymbol{\Gamma}_{1}-\boldsymbol{\Gamma}_{2}\right) /\left(\omega_{1}-\omega_{2}\right)$. For a strongly hierarchical spectrum the last two terms are negligible and (5.19) reverts to (5.17a). On the other hand, for a quasi-degenerate mass 
C-violation in runaway regime, $\mathrm{R}=1$

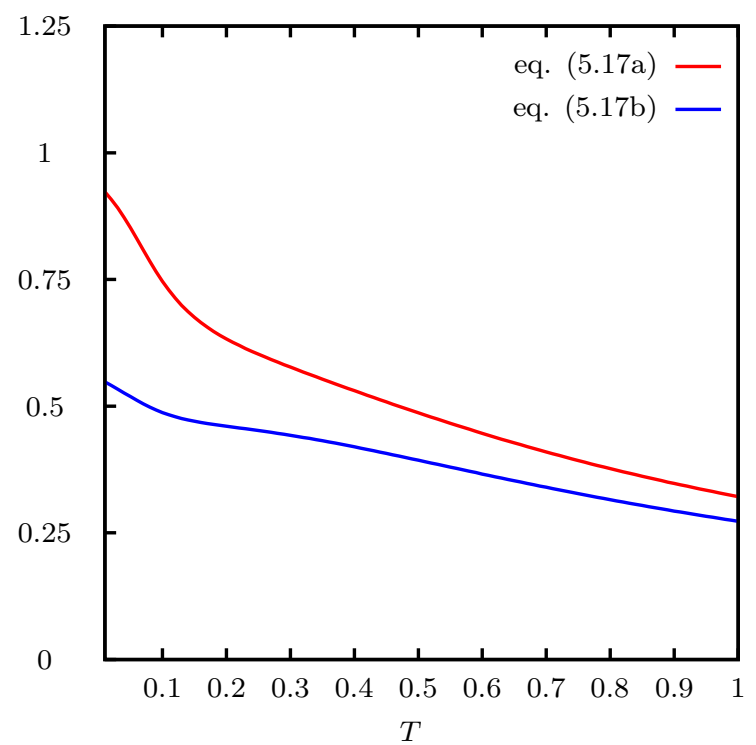

C-violation in crossing regime, $\mathrm{R}=1$

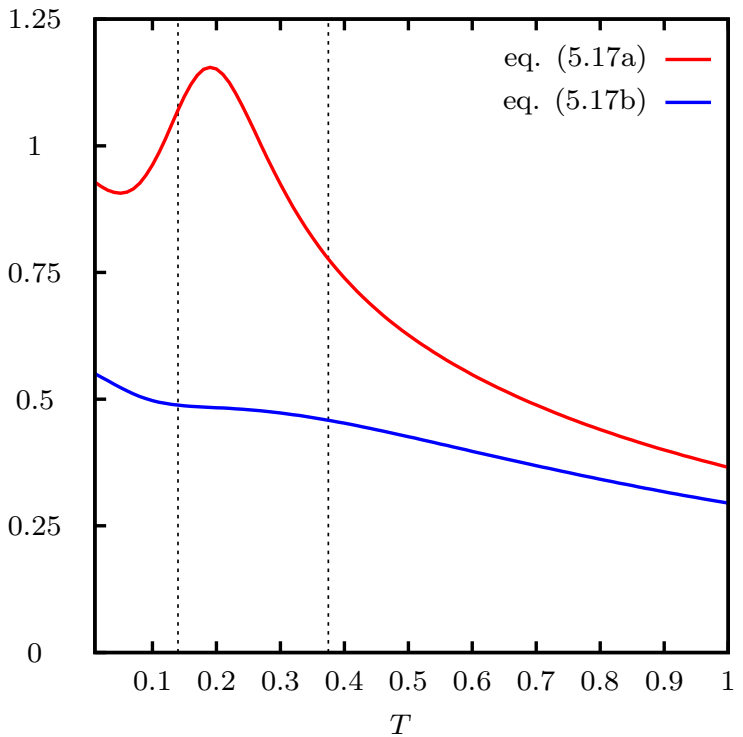

Figure 5. Dependence of the asymptotic value of $\operatorname{tr} \eta$ (in units of $1 / \mu$ ) on the temperature (in units of $\mu$ ) in the runaway and crossing regimes for $R=1$.

spectrum we can neglect the difference between $\omega_{2}$ and $\omega_{1}$ and replace them by $\bar{\omega}$ in $\Pi_{\rho}$ and $\Pi_{h}$. The $\delta$-terms then cancel out, the last line vanishes and (5.19) reverts to (5.17b). The $R$ dependence of $\operatorname{tr} \eta$ for various values of the temperature is presented in figure 6 . In both regimes $\operatorname{tr} \eta$ vanishes at very large and very small values of the degeneracy parameter because for large $R$ the $C$-violating parameter is proportional to $1 / R$ whereas for small $R$ it is proportional to $R$. Let us stress once again that $\operatorname{tr} \eta$ automatically vanishes in the limit $M_{2}=M_{1}$, i.e. for $R=0$, because the Lagrangian is $C$-symmetric in this case. Just as expected, eq. (5.19) gives an accurate result for the final asymmetry not only for a hierarchical or quasi-degenerate spectrum, but also for the intermediate values of $R$. On the other hand, the expression obtained assuming a hierarchical mass spectrum, eq. (5.17a), overestimates the final asymmetry at small $R$, whereas the expression obtained assuming a quasi-degenerate mass spectrum overestimates the final asymmetry at large $R$. In the runaway regime both the hierarchical and quasi-degenerate approximations are smooth for all values of $R$ and give similar results. On the other hand, in the crossing regime the hierarchical approximation for $\operatorname{tr} \eta$ develops a spurious peak that we have already observed at figure 5. The contribution of the negative interference terms ensures that the exact result for $\operatorname{tr} \eta$ remains smooth for all values of $R$ and $T$ and does not develop a peak in the vicinity of the crossing point.

\section{$6 \quad$ Numerical treatment of sub-leading effects}

In this section we compare the contribution of off-shell effects in the late time limit by computing numerically the contribution of the second, third and fourth terms in (5.2). Thereby we show that the assumptions made in the previous section are justified. To this 

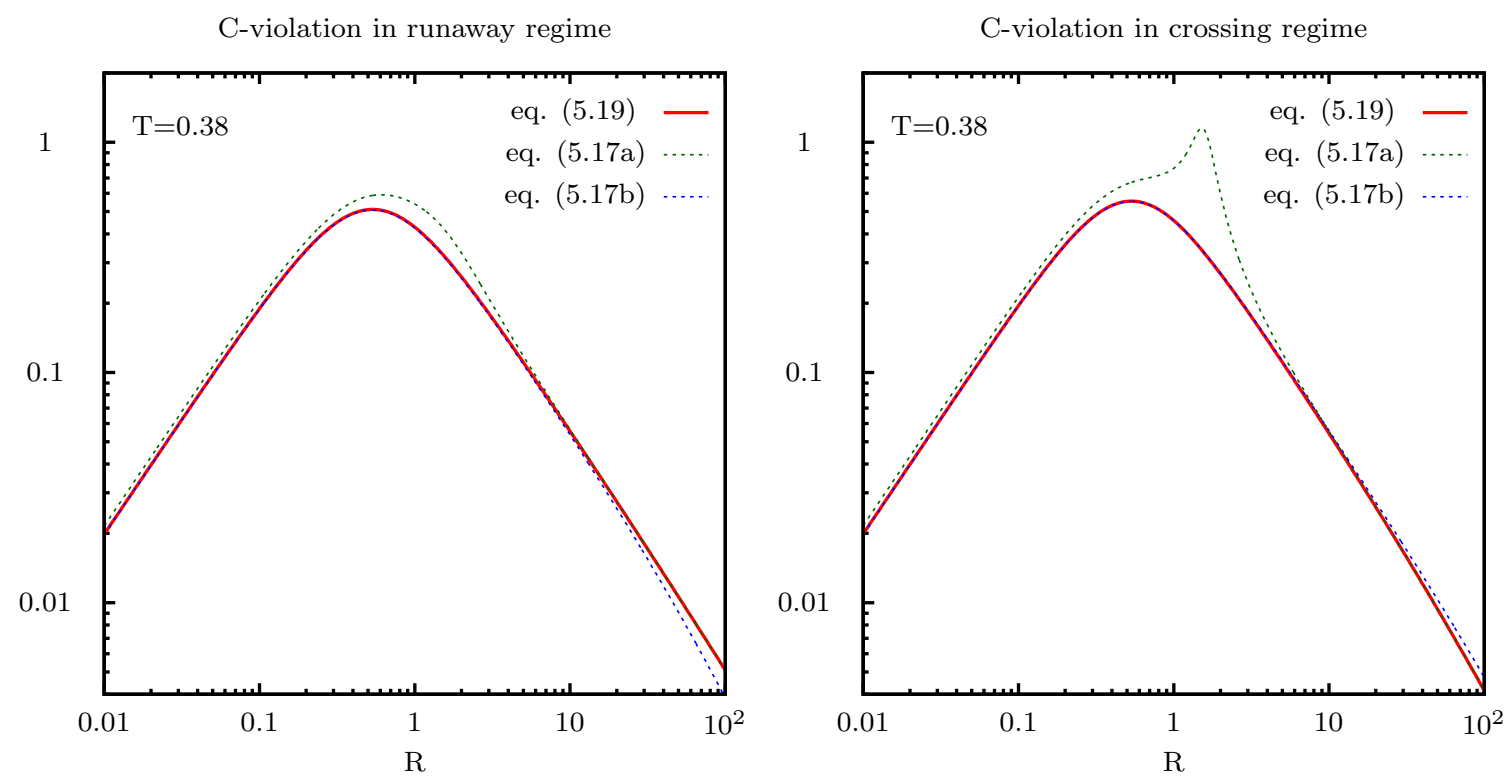

Figure 6. Dependence of the asymptotic value of $\operatorname{tr} \eta$ (in units of $1 / \mu$ ) on the degeneracy parameter $R$ in the runaway and crossing regimes (in units of $\mu$ ).

end we rewrite (5.2), using (5.1), as

$$
\operatorname{tr} \eta(t, \mathbf{q})=-\frac{2 J}{\operatorname{det} M} \int_{0}^{\infty} \frac{\Pi_{\rho}\left(q_{0}, \mathbf{q}\right)}{(2 \pi)^{3}} \operatorname{Im}\left(I_{1} I_{2}-I_{3} I_{4}+I_{1} I_{5}-I_{3} I_{6}\right) d q_{0},
$$

with

$$
\begin{array}{lll}
I_{1}=\int_{0}^{\infty} d p_{0} \frac{\left(1-e^{i\left(q_{0}-p_{0}\right) t}\right) \Pi_{R}\left(p_{0}, \mathbf{q}\right)}{\left(q_{0}-p_{0}\right) \operatorname{det} \Omega_{R}\left(p_{0}, \mathbf{q}\right)}, & I_{2}=\int_{0}^{\infty} d k_{0} \frac{1-e^{-i\left(q_{0}-k_{0}\right) t}}{\left(q_{0}-k_{0}\right) \operatorname{det} \Omega_{A}\left(k_{0}, \mathbf{q}\right)}, \\
I_{3}=\int_{0}^{\infty} d p_{0} \frac{\left(1-e^{-i\left(q_{0}+p_{0}\right) t}\right) \Pi_{R}\left(p_{0}, \mathbf{q}\right)}{\left(q_{0}+p_{0}\right) \operatorname{det} \Omega_{R}\left(p_{0}, \mathbf{q}\right)}, & I_{4}=\int_{0}^{\infty} d k_{0} \frac{1-e^{i\left(q_{0}+k_{0}\right) t}}{\left(q_{0}+k_{0}\right) \operatorname{det} \Omega_{A}\left(k_{0}, \mathbf{q}\right)}, \\
I_{5}=\int_{0}^{\infty} d p_{0} \frac{\left(1-e^{-i\left(q_{0}-p_{0}\right) t}\right)}{\left(q_{0}+p_{0}\right) \operatorname{det} \Omega_{R}\left(p_{0}, \mathbf{q}\right)}, & I_{6}=\int_{0}^{\infty} d k_{0} \frac{1-e^{i\left(q_{0}-k_{0}\right) t}}{\left(q_{0}-k_{0}\right) \operatorname{det} \Omega_{A}\left(k_{0}, \mathbf{q}\right)} .
\end{array}
$$

The different integrals $I_{1} \ldots I_{6}$ are all complex valued and finite. In particular the integrands are finite at $q_{0}=p_{0}, k_{0}$. The integrands of $I_{1}, I_{3}, I_{5}$ exhibit integrable singularities for $p_{0}=|\mathbf{q}|$ due to logarithmic singularities of the self-energies. As discussed below equation (5.2), the contribution of the first term dominates the total of (6.1) and setting the last three terms in $I_{3} \ldots I_{6}$ to zero would correspond to the approximation used in the previous section. Here we are interested in their relative contribution in order to know how accurate our assumptions were.

At finite time the contributions to the integrands by the exponentials are oscillating as functions of the integration variables (with frequency $t$ ). Since the integrals extend to $+\infty$, a large simplification is achieved if we restrict ourselves to the late-time limit. Similar to (5.18), it is obtained by substituting $q_{0} \rightarrow q_{0} \pm i \epsilon$ in each of the integrals. We obtain 
Quality of Breit-Wigner approximation, $\mathrm{T}=1$

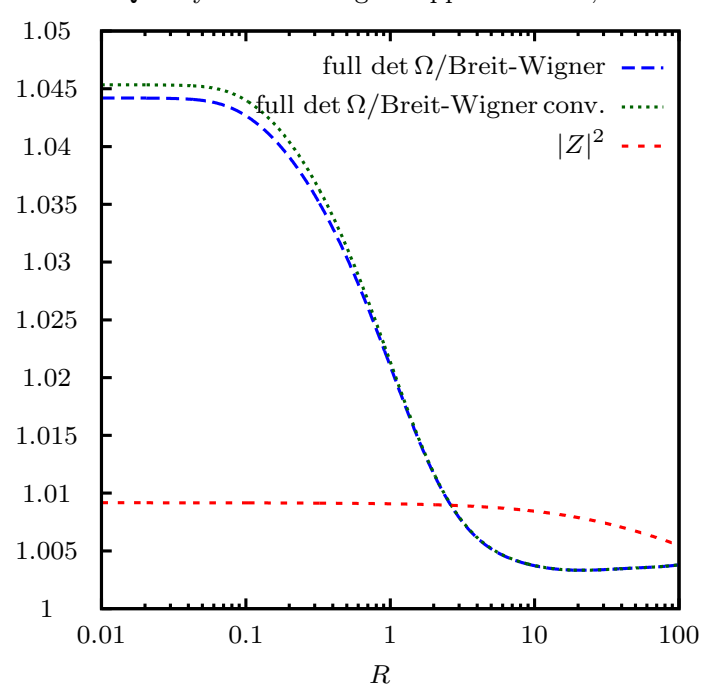

Figure 7. Dependence of the ratio of $\operatorname{tr} \eta(\infty, \mathbf{q})$, computed without Breit-Wigner approximation, on the degeneracy parameter in the crossing regime for $T=1$ (in units of $\mu$ ). Also shown is the value of $|Z|$ which enters the result in the Breit-Wigner approximation. The $R$-dependence in the runaway regime is similar and not shown. 'Breit-Wigner conv.' refers to the conventional method to determine effective masses and widths sketched above in which one searches the complex zeros of $\operatorname{det} \Omega$.

for $t \rightarrow \infty$ :

$$
\begin{array}{lll}
I_{1}=\lim _{\epsilon \rightarrow 0} \int_{0}^{\infty} \frac{\Pi_{R}\left(p_{0}, \mathbf{q}\right) d p_{0}}{\left(q_{0}-p_{0}+i \epsilon\right) \operatorname{det} \Omega_{R}\left(p_{0}, \mathbf{q}\right)}, & I_{2}=\lim _{\epsilon \rightarrow 0} \int_{0}^{\infty} \frac{d k_{0}}{\left(q_{0}-k_{0}-i \epsilon\right) \operatorname{det} \Omega_{A}\left(k_{0}, \mathbf{q}\right)}, \\
I_{3}=\int_{0}^{\infty} \frac{\Pi_{R}\left(p_{0}, \mathbf{q}\right) d p_{0}}{\left(q_{0}+p_{0}\right) \operatorname{det} \Omega_{R}\left(p_{0}, \mathbf{q}\right)}, & I_{4}=\int_{0}^{\infty} \frac{d k_{0}}{\left(q_{0}+k_{0}\right) \operatorname{det} \Omega_{A}\left(k_{0}, \mathbf{q}\right)}, \\
I_{5}=\int_{0}^{\infty} \frac{d p_{0}}{\left(q_{0}+p_{0}\right) \operatorname{det} \Omega_{R}\left(p_{0}, \mathbf{q}\right)}, & I_{6}=\lim _{\epsilon \rightarrow 0} \int_{0}^{\infty} \frac{d k_{0}}{\left(q_{0}-k_{0}+i \epsilon\right) \operatorname{det} \Omega_{A}\left(k_{0}, \mathbf{q}\right)} .
\end{array}
$$

Technically we choose a small constant value for $\epsilon$ in the numerical computation which we decrease until the results do not change significantly anymore. The performed transformations have turned (6.1) into a two-fold integral. However the hermitian self-energy included in $\Pi_{R}$ contains a further integral such that the overall dimensionality of $\operatorname{tr} \eta(t, \mathbf{q})$ is 3 . The quadrature is complicated by the presence of the integrable singularity of $\Pi_{R}$ at $p_{0}=|\mathbf{q}|$ and the poles due to $\operatorname{det} \Omega_{R}$ and $\operatorname{det} \Omega_{A}$. These difficulties can be overcome by performing appropriate integral transformations on small intervals surrounding these poles.

Further simplification is achieved by performing the Breit-Wigner approximation in (6.3), i.e. by using (5.3) and (5.4) everywhere. In this approximation it is possible to obtain closed-form analytic expressions for $I_{1} \ldots I_{6}$ neglecting the finite temperature contributions to $\Pi_{R}$. At non-zero temperature these contributions to $I_{1}$ and $I_{3}$ still need to be integrated numerically but the partial analytic results stabilize the computation of the total integral (6.1). To demonstrate the accuracy of the Breit-Wigner approximation we present the ratio of the first term of (6.1), computed without and with this assumption, in figure 7 . We find that the relative error made for average momenta is below $5 \%$, even for smallest degeneracy parameters. 
Off-shell contributions in runaway regime, $\mathrm{R}=1$

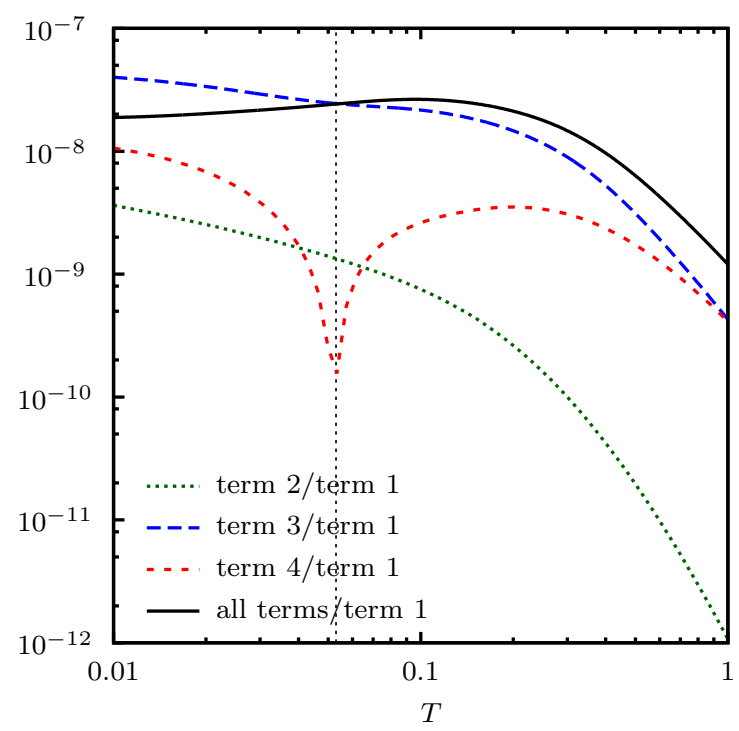

Off-shell contributions in crossing regime, $\mathrm{R}=1$

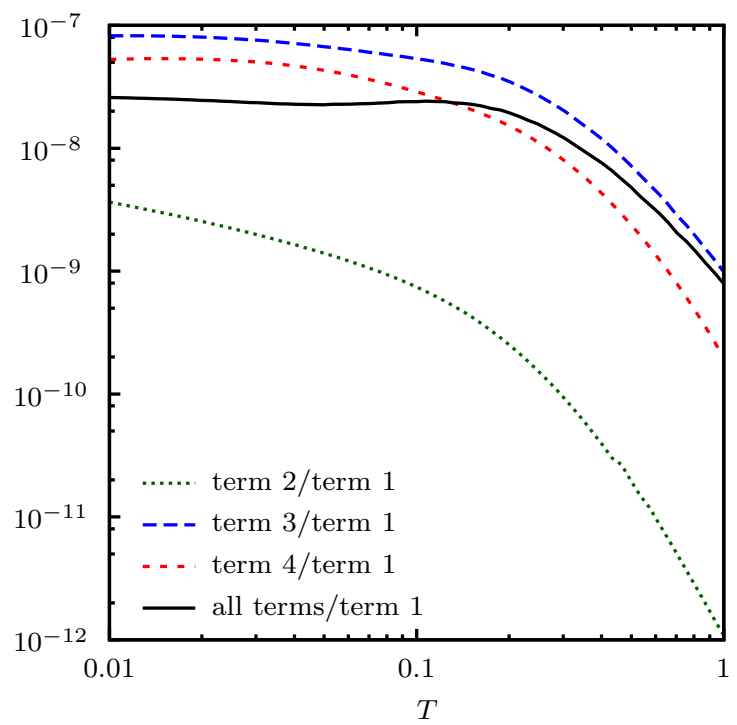

Figure 8. Ratio of the asymptotic values of the contributions of the second, third and fourth term of (5.2) to that of the the first term. Shown is the dependence on the temperature (in units of $\mu$ ) in the runaway and crossing regimes for $R=1$. The contributions from term 3 and term 4 have opposite sign for $T$ below the dotted vertical line.

This result motivates us to study the contribution of off-shell effects in the Breit-Wigner approximation as well. Figure 8 shows the $T$-dependence of the relative contributions by the second, third and fourth term to (6.1) with respect to that by the first one. The contributions of the third and fourth terms are small, for all values of $T$, compared to the onshell contribution because only one of the $\left(q_{0} \pm k_{0}\right)$ and $\left(q_{0} \pm p_{0}\right)$ factors in the denominators of the integrands of $I_{1}, I_{3}, I_{5}, I_{6}$ can vanish for each of them. For the same reason the contribution of the second term is even smaller. In this case none of the $\left(q_{0}+k_{0}\right)$ and $\left(q_{0}+p_{0}\right)$ terms can vanish since the integration is only over positive momenta. Additionally the two contributions by the third and the fourth term enter with opposite signs (only for small $T$ in case of the runaway regime).

Figure 9 shows the $R$-dependence of the same quantities for fixed temperature $T=1$ (in units of $\mu$ ) and the corresponding average momentum q. Remarkably the relative contribution of off-shell effects increases with increasing degeneracy parameter. It flattens for large $R$ and stays below $1 \%$ in our examples. For this behaviour it is crucial that the contributions of the third and fourth terms of (6.1) cancel again for large $R$.

\section{Summary and outlook}

For resonant leptogenesis the mass difference is typically of the order of the sum of the decay widths. Therefore the thermal corrections to the effective masses, which are of the order of the widths, are comparable to the mass difference. Depending on the values of the couplings either runway or crossing regime is realized. These have not been discussed in the context of leptogenesis before. In the runaway regime the mass difference grows with increasing temperature, whereas in the crossing regime the difference of the masses 
Off-shell contributions in runaway regime, $\mathrm{T}=1$

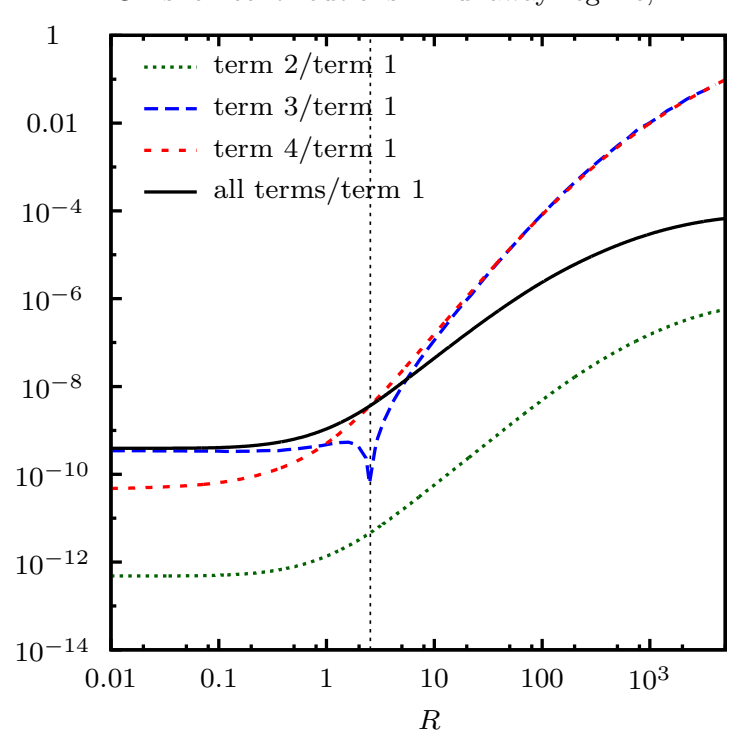

Off-shell contributions in crossing regime, $\mathrm{T}=1$

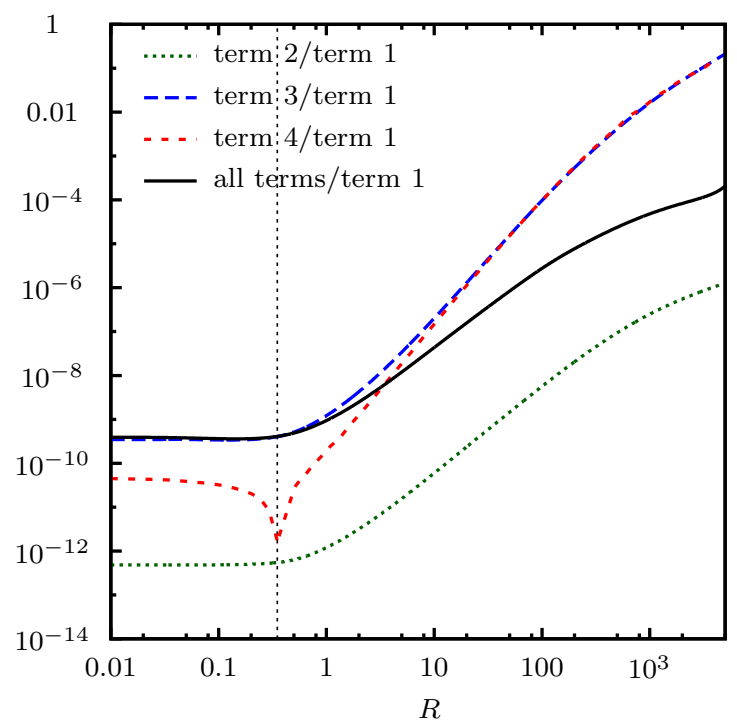

Figure 9. Ratio of the asymptotic values of the contributions of the second, third and fourth term of (5.2) and that of the the first term. Shown is the dependence on the degeneracy parameter in the runaway and crossing regimes for $T / \mu=1$. The contributions from term 3 and term 4 have opposite sign for $R$ above the dotted vertical line.

initially decreases, such that the effective masses become equal at some temperature, and then increase again at even higher $T$. The main goal of this work was to investigate the asymmetry generation in the vicinity of the crossing point. We obtained consistent results for the $C P$-violating source term which maintains the $C P$-properties of the Lagrangian as the temperature changes. In particular this enabled us to find answers to three questions which may be asked based on the results of previous studies relying on Boltzmann-like equations combined with conventional quantum field theory: is the source term suppressed (or does it even change sign) when the difference of the effective masses vanishes at the crossing point? Can a Boltzmann-like approximation, which takes into account thermal corrections to the masses and decay widths, adequately describe the asymmetry generation close to a crossing point? What is the relative size of off-shell contributions beyond the Breit-Wigner approximation?

As far as the first question is concerned, it has been found in earlier works that the $C P$-violating parameters are proportional to the mass difference. In the limit of equal masses they vanish (as the mass difference passes through zero) which is consistent with the $C P$-invariance of the Lagrangian in this case. Because the difference of the effective masses vanishes at the crossing temperature, one could naively expect that $C P$-violation also vanishes or is at least suppressed at the crossing point. Here we have demonstrated analytically that the masses in the numerator and denominator of (1.1) have different origin. Whereas masses and widths in the denominator of the canonical expression for the $C P$-violating parameter may be interpreted as the effective thermal masses, the numerator contains the mass parameters of the Lagrangian. These do not depend on temperature by definition. Therefore, contrary to the naive expectation, the vanishing of the difference of 
the effective masses by no means implies vanishing of the $C P$-violating source term. The ability to distinguish between the mass parameters of the Lagrangian and the effective masses relies on an important technical aspect of our analysis. In contrast to the approach followed in earlier works we did not use renormalization group equations to minimize the difference between the mass parameters and effective masses at each temperature. Instead we have fixed the renormalization scale at zero temperature. As a result, the mass matrix appearing in the Kadanoff-Baym equations and throughout the rest of the paper coincides with the mass parameters of the Lagrangian. The latter can in principle be measured experimentally at zero temperature in e.g. decay and scattering experiments. In other words, this approach has the advantage that the relation between the, in principle measurable, zero-temperature masses and widths and the asymmetry generated at temperatures comparable to the masses remains transparent.

Concerning the second question, peaks of the spectral functions that correspond to the quasiparticle excitations may strongly overlap in the resonant regime. This renders the applicability of the Boltzmann approximation questionable. One could expect that this approximation breaks down completely at the crossing point. Our analysis confirms that even taking the thermal effects in the form of effective masses and widths into account does not substantially improve the quality of the quasiparticle approximation. In particular, close to the crossing point the asymmetry computed in the Boltzmann approximation develops a spurious peak absent in the exact result. Additional contributions that describe coherent transitions between the two mass shells exactly compensate the enhancement of the non-oscillating contributions. We would like to stress that these coherent transitions are determined by basis-invariant effective masses and widths and affect all components of the two-point functions, which are not basis-invariant. In other words, the dynamics is basiscovariant and can be formulated in terms of basis-invariant quantities. Because in both regimes the mass difference grows at high temperatures one can expect that the quality of quasiparticle approximation increases. We find that this is indeed the case. However, this improvement is not related to an increasing separation of the peaks of the spectral function. It turns out that for a quasi-degenerate mass spectrum at high temperatures the positions of the peaks are not determined by the effective masses.

Finally, coming to the third question, the very fact that in the crossing regime the quasiparticle approximation completely breaks down also raises the question of the relative size of off-shell contributions. Comparing our analytical results with exact numerical computations we find that purely off-shell effects are small, of the order of $1 \%$ or less for our benchmark scenarios. The Breit-Wigner approximation itself entails a relative error of less than $5 \%$ even at the crossing point. Note that all our computations relied on the toy-model and that quantitative results could differ for the phenomenological scenario.

In addition to clarifying the qualitatively important and interesting questions raised above, the results of this work also provide a reference solution for various approximation schemes. Here we have demonstrated that the Boltzmann approximation is not applicable in the crossing regime. In a forthcoming publication we will perform a more detailed comparison of the Kadanoff-Baym and density matrix equations and present a derivation of the density matrix equations applicable for quasidegenerate as well as hierarchical mass spectra from first-principles. 


\section{Acknowledgments}

We would like to thank M. Shaposhnikov for valuable discussions. This research was supported in part by the National Science Foundation under Grant No. PHY11-25915.

Open Access. This article is distributed under the terms of the Creative Commons Attribution License (CC-BY 4.0), which permits any use, distribution and reproduction in any medium, provided the original author(s) and source are credited.

\section{References}

[1] M. Fukugita and T. Yanagida, Baryogenesis Without Grand Unification, Phys. Lett. B 174 (1986) 45 [INSPIRE].

[2] E.K. Akhmedov, V.A. Rubakov and A.Y. Smirnov, Baryogenesis via neutrino oscillations, Phys. Rev. Lett. 81 (1998) 1359 [hep-ph/9803255] [INSPIRE].

[3] M.A. Luty, Baryogenesis via leptogenesis, Phys. Rev. D 45 (1992) 455 [inSPIRE].

[4] M. Flanz, E.A. Paschos and U. Sarkar, Baryogenesis from a lepton asymmetric universe, Phys. Lett. B 345 (1995) 248 [Erratum ibid. B 382 (1996) 447] [hep-ph/9411366] [INSPIRE].

[5] L. Covi, E. Roulet and F. Vissani, CP violating decays in leptogenesis scenarios, Phys. Lett. B 384 (1996) 169 [hep-ph/9605319] [INSPIRE].

[6] E. Roulet, L. Covi and F. Vissani, On the CP asymmetries in Majorana neutrino decays, Phys. Lett. B 424 (1998) 101 [hep-ph/9712468] [INSPIRE].

[7] S. Davidson and A. Ibarra, A lower bound on the right-handed neutrino mass from leptogenesis, Phys. Lett. B 535 (2002) 25 [hep-ph/0202239] [INSPIRE].

[8] W. Buchmüller, P. Di Bari and M. Plümacher, Cosmic microwave background, matter-antimatter asymmetry and neutrino masses, Nucl. Phys. B 643 (2002) 367 [Erratum ibid. B 793 (2008) 362] [hep-ph/0205349] [INSPIRE].

[9] W. Buchmüller, P. Di Bari and M. Plümacher, The neutrino mass window for baryogenesis, Nucl. Phys. B 665 (2003) 445 [hep-ph/0302092] [inSPIRE].

[10] P. Di Bari, Leptogenesis, neutrino mixing data and the absolute neutrino mass scale, hep-ph/0406115 [INSPIRE].

[11] A. Abada et al., Flavour Matters in Leptogenesis, JHEP 09 (2006) 010 [hep-ph/0605281] [INSPIRE].

[12] S. Blanchet and P. Di Bari, Flavor effects in thermal leptogenesis, Nucl. Phys. Proc. Suppl. 168 (2007) 372 [hep-ph/0702089] [INSPIRE].

[13] F.X. Josse-Michaux and A. Abada, Study of flavour dependencies in leptogenesis, JCAP 10 (2007) 009 [hep-ph/0703084] [INSPIRE].

[14] S. Blanchet and P. Di Bari, New aspects of leptogenesis bounds, Nucl. Phys. B 807 (2009) 155 [arXiv:0807.0743] [INSPIRE].

[15] L. Covi, N. Rius, E. Roulet and F. Vissani, Finite temperature effects on CP-violating asymmetries, Phys. Rev. D 57 (1998) 93 [hep-ph/9704366] [INSPIRE]. 
[16] G.F. Giudice, A. Notari, M. Raidal, A. Riotto and A. Strumia, Towards a complete theory of thermal leptogenesis in the SM and MSSM, Nucl. Phys. B 685 (2004) 89 [hep-ph/0310123] [INSPIRE].

[17] C.P. Kiessig and M. Plümacher, Thermal masses in leptogenesis, AIP Conf. Proc. 1200 (2010) 999 [arXiv: 0910.4872] [INSPIRE].

[18] M. Garny, A. Hohenegger and A. Kartavtsev, Medium corrections to the CP-violating parameter in leptogenesis, Phys. Rev. D 81 (2010) 085028 [arXiv:1002.0331] [INSPIRE].

[19] M. Shaposhnikov, The nuMSM, leptonic asymmetries and properties of singlet fermions, JHEP 08 (2008) 008 [arXiv:0804.4542] [INSPIRE].

[20] A. Boyarsky, O. Ruchayskiy and M. Shaposhnikov, The role of sterile neutrinos in cosmology and astrophysics, Ann. Rev. Nucl. Part. Sci. 59 (2009) 191 [arXiv:0901.0011] [InSPIRE].

[21] M. Shaposhnikov, Baryon asymmetry of the universe and neutrinos, Prog. Theor. Phys. 122 (2009) 185 [INSPIRE].

[22] L. Canetti and M. Shaposhnikov, Baryon Asymmetry of the Universe in the NuMSM, JCAP 09 (2010) 001 [arXiv: 1006.0133] [INSPIRE].

[23] T. Asaka, S. Eijima and H. Ishida, Kinetic Equations for Baryogenesis via Sterile Neutrino Oscillation, JCAP 02 (2012) 021 [arXiv:1112.5565] [INSPIRE].

[24] L. Canetti, M. Drewes and M. Shaposhnikov, Sterile Neutrinos as the Origin of Dark and Baryonic Matter, Phys. Rev. Lett. 110 (2013) 061801 [arXiv:1204.3902] [INSPIRE].

[25] L. Canetti, M. Drewes and M. Shaposhnikov, Matter and Antimatter in the Universe, New J. Phys. 14 (2012) 095012 [arXiv: 1204.4186] [INSPIRE].

[26] B. Shuve and I. Yavin, Baryogenesis through Neutrino Oscillations: A Unified Perspective, Phys. Rev. D 89 (2014) 075014 [arXiv: 1401.2459] [InSPIRE].

[27] P.S.B. Dev, P. Millington, A. Pilaftsis and D. Teresi, Flavour Covariant Transport Equations: an Application to Resonant Leptogenesis, arXiv:1404.1003 [INSPIRE].

[28] G. Sigl and G. Raffelt, General kinetic description of relativistic mixed neutrinos, Nucl. Phys. B 406 (1993) 423 [INSPIRE].

[29] J.-S. Gagnon and M. Shaposhnikov, Baryon Asymmetry of the Universe without Boltzmann or Kadanoff-Baym equations, Phys. Rev. D 83 (2011) 065021 [arXiv:1012.1126] [INSPIRE].

[30] W. Buchmüller and S. Fredenhagen, Quantum mechanics of baryogenesis, Phys. Lett. B 483 (2000) 217 [hep-ph/0004145] [INSPIRE].

[31] A. Anisimov, W. Buchmüller, M. Drewes and S. Mendizabal, Nonequilibrium Dynamics of Scalar Fields in a Thermal Bath, Annals Phys. 324 (2009) 1234 [arXiv:0812.1934] [INSPIRE].

[32] A. Anisimov, W. Buchmüller, M. Drewes and S. Mendizabal, Quantum Leptogenesis I, Annals Phys. 326 (2011) 1998 [Erratum ibid. 338 (2011) 376-377] [arXiv:1012.5821] [INSPIRE].

[33] A. Anisimov, W. Buchmüller, M. Drewes and S. Mendizabal, Leptogenesis from Quantum Interference in a Thermal Bath, Phys. Rev. Lett. 104 (2010) 121102 [arXiv:1001.3856] [INSPIRE]. 
[34] M. Garny, A. Hohenegger, A. Kartavtsev and M. Lindner, Systematic approach to leptogenesis in nonequilibrium QFT: Vertex contribution to the CP-violating parameter, Phys. Rev. D 80 (2009) 125027 [arXiv:0909.1559] [INSPIRE].

[35] M. Garny, A. Hohenegger, A. Kartavtsev and M. Lindner, Systematic approach to leptogenesis in nonequilibrium QFT: Self-energy contribution to the CP-violating parameter, Phys. Rev. D 81 (2010) 085027 [arXiv:0911.4122] [INSPIRE].

[36] M. Beneke, B. Garbrecht, M. Herranen and P. Schwaller, Finite Number Density Corrections to Leptogenesis, Nucl. Phys. B 838 (2010) 1 [arXiv:1002.1326] [INSPIRE].

[37] B. Garbrecht, Leptogenesis: The Other Cuts, Nucl. Phys. B 847 (2011) 350 [arXiv: 1011.3122] [INSPIRE].

[38] T. Frossard, M. Garny, A. Hohenegger, A. Kartavtsev and D. Mitrouskas, Systematic approach to thermal leptogenesis, Phys. Rev. D 87 (2013) 085009 [arXiv:1211.2140] [INSPIRE].

[39] T. Frossard, A. Kartavtsev and D. Mitrouskas, Systematic approach to $\Delta L=1$ processes in thermal leptogenesis, Phys. Rev. D 87 (2013) 125006 [arXiv:1304.1719] [INSPIRE].

[40] A. De Simone and A. Riotto, Quantum Boltzmann Equations and Leptogenesis, JCAP 08 (2007) 002 [hep-ph/0703175] [INSPIRE].

[41] M. Beneke, B. Garbrecht, C. Fidler, M. Herranen and P. Schwaller, Flavoured Leptogenesis in the CTP Formalism, Nucl. Phys. B 843 (2011) 177 [arXiv:1007.4783] [InSPIRE].

[42] M. Drewes and B. Garbrecht, Leptogenesis from a GeV Seesaw without Mass Degeneracy, JHEP 03 (2013) 096 [arXiv:1206.5537] [INSPIRE].

[43] B. Garbrecht and M. Herranen, Effective Theory of Resonant Leptogenesis in the Closed-Time-Path Approach, Nucl. Phys. B 861 (2012) 17 [arXiv:1112.5954] [InSPIRE].

[44] M. Flanz, E.A. Paschos, U. Sarkar and J. Weiss, Baryogenesis through mixing of heavy Majorana neutrinos, Phys. Lett. B 389 (1996) 693 [hep-ph/9607310] [INSPIRE].

[45] L. Covi and E. Roulet, Baryogenesis from mixed particle decays, Phys. Lett. B 399 (1997) 113 [hep-ph/9611425] [INSPIRE].

[46] A. Pilaftsis, CP violation and baryogenesis due to heavy Majorana neutrinos, Phys. Rev. D 56 (1997) 5431 [hep-ph/9707235] [INSPIRE].

[47] A. Pilaftsis, Resonant CP-violation induced by particle mixing in transition amplitudes, Nucl. Phys. B 504 (1997) 61 [hep-ph/9702393] [INSPIRE].

[48] A. Pilaftsis, Heavy Majorana neutrinos and baryogenesis, Int. J. Mod. Phys. A 14 (1999) 1811 [hep-ph/9812256] [INSPIRE].

[49] W. Buchmüller and M. Plümacher, Neutrino masses and the baryon asymmetry, Int. J. Mod. Phys. A 15 (2000) 5047 [hep-ph/0007176] [InSPIRE].

[50] A. Pilaftsis and T.E.J. Underwood, Resonant leptogenesis, Nucl. Phys. B 692 (2004) 303 [hep-ph/0309342] [INSPIRE].

[51] A. Pilaftsis and T.E.J. Underwood, Electroweak-scale resonant leptogenesis, Phys. Rev. D 72 (2005) 113001 [hep-ph/0506107] [INSPIRE].

[52] A. Anisimov, A. Broncano and M. Plümacher, The CP-asymmetry in resonant leptogenesis, Nucl. Phys. B 737 (2006) 176 [hep-ph/0511248] [INSPIRE]. 
[53] S. Blanchet, Z. Chacko, S.S. Granor and R.N. Mohapatra, Probing Resonant Leptogenesis at the LHC, Phys. Rev. D 82 (2010) 076008 [arXiv:0904.2174] [InSPIRE].

[54] A. Ibarra, E. Molinaro and S.T. Petcov, Low Energy Signatures of the TeV Scale See-Saw Mechanism, Phys. Rev. D 84 (2011) 013005 [arXiv: 1103.6217] [InSPIRE].

[55] P.S.B. Dev, A. Pilaftsis and U.-k. Yang, New Production Mechanism for Heavy Neutrinos at the LHC, Phys. Rev. Lett. 112 (2014) 081801 [arXiv: 1308.2209] [INSPIRE].

[56] F.F. Deppisch, J. Harz and M. Hirsch, Falsifying High-Scale Leptogenesis at the LHC, Phys. Rev. Lett. 112 (2014) 221601 [arXiv:1312.4447] [INSPIRE].

[57] L. Canetti, M. Drewes and B. Garbrecht, Lab-to-Genesis, arXiv:1404.7114 [INSPIRE].

[58] A. Das, P.S. Bhupal Dev and N. Okada, Direct Bounds on Electroweak Scale Pseudo-Dirac Neutrinos from $\sqrt{s}=8 \mathrm{TeV}$ LHC Data, arXiv:1405.0177 [INSPIRE].

[59] W. Bonivento et al., Proposal to Search for Heavy Neutral Leptons at the SPS, arXiv:1310.1762 [INSPIRE].

[60] B.A. Kniehl and A. Pilaftsis, Mixing renormalization in Majorana neutrino theories, Nucl. Phys. B 474 (1996) 286 [hep-ph/9601390] [INSPIRE].

[61] A. Hohenegger and A. Kartavtsev, Basis invariant measure of CP-violation and renormalization, arXiv:1309.1385 [INSPIRE].

[62] M. Garny, A. Kartavtsev and A. Hohenegger, Leptogenesis from first principles in the resonant regime, Annals Phys. 328 (2013) 26 [arXiv:1112.6428] [INSPIRE].

[63] M. Garny, A. Hohenegger and A. Kartavtsev, Quantum corrections to leptogenesis from the gradient expansion, arXiv: 1005.5385 [INSPIRE].

[64] S. Iso, K. Shimada and M. Yamanaka, Kadanoff-Baym approach to the thermal resonant leptogenesis, JHEP 04 (2014) 062 [arXiv: 1312.7680] [INSPIRE].

[65] S. Iso and K. Shimada, Coherent Flavour Oscillation and CP-violating Parameter in Thermal Resonant Leptogenesis, arXiv:1404.4816 [INSPIRE].

[66] V. Cirigliano, C. Lee, M.J. Ramsey-Musolf and S. Tulin, Flavored Quantum Boltzmann Equations, Phys. Rev. D 81 (2010) 103503 [arXiv:0912.3523] [INSPIRE]. 